\title{
Synthesis and Properties of Aza-Ovalene with Six Zigzag Edges
}

(Supporting Information)

Laiyun Zhou, ${ }^{\dagger}$ Boming Wu, ${ }^{\dagger}$ Yuanyuan Chen,${ }^{\dagger}$ Jianye, Gong, ${ }^{\dagger}$ Jianguo Wang, ${ }^{\dagger}$ Gaole Dai, ${ }^{*}$ Chunyan Chi, ${ }^{*}$ Qing Wang, ${ }^{* \dagger}$

${ }^{\dagger}$ School of Chemistry and Chemical Engineering, Inner Mongolia University, 235

West University Street, Hohhot 010021, China

Email:qingwang@imu.edu.cn

* Institute of Functional Nano \& Soft Materials (FUNSOM), Jiangsu Key Laboratory for Carbon-Based Functional Materials \& Devices, Soochow University, 199 Ren'ai Road, Suzhou, Jiangsu 215123, China

Email:daigaole@suda.edu.cn

$\S$ Department of Chemistry, National University of Singapore, 3 Science Drive 3, 117543, Singapore

Email:.chmcc@nus.edu.sg

\section{Table of Contents}

1. Experimental Section........................................................................................ 2

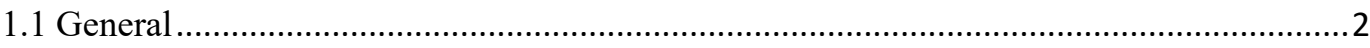

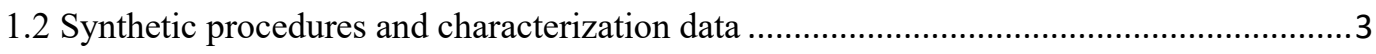

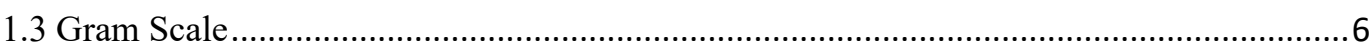

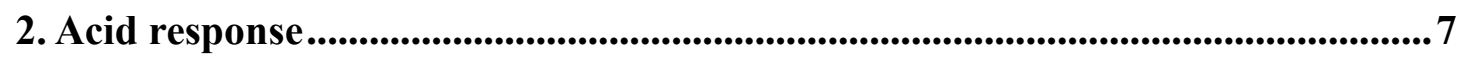

3. Cyclic voltammetry ..............................................................................................10

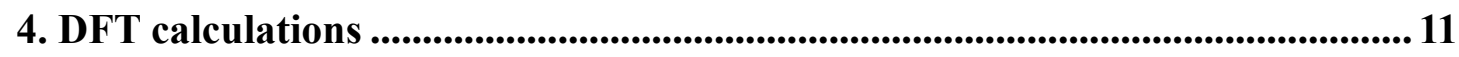

5. Appendix I: X-ray crystallographic data....................................................................20

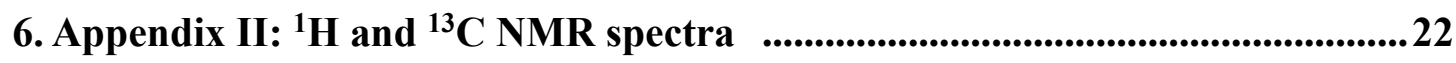

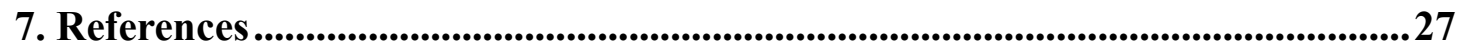




\section{Experimental Section}

\subsection{General}

All reagents were purchased from commercial sources without further purification. Anhydrous THF were distilled from sodium-benzophenone immediately prior to use. ${ }^{1} \mathrm{H}$ and ${ }^{13} \mathrm{C}$ NMR spectra were recorded using Bruker Advance II $500 \mathrm{MHz}$ spectrometer in $\mathrm{CDCl}_{3}$ with tetramethylsilane (TMS) as the internal standard. The chemical shift was recorded in ppm and the following abbreviations were used to explain the multiplicities: $\mathrm{s}=$ singlet, $\mathrm{d}=$ doublet, $\mathrm{t}=$ triplet, $\mathrm{m}=$ multiplet, $\mathrm{br}=$ broad. MALDI-TOF mass spectra were recorded on a MicrOTOFQII instrument. UV-Vis absorption was recorded on a SHIMADZU UV-2600i spectrophotometer. Cyclic voltammetry measurements were performed in dry dichloromethane on a $\mathrm{CHI} 620 \mathrm{C}$ electrochemical analyzer with a three-electrode cell, using $0.1 \mathrm{M} \mathrm{Bu}_{4} \mathrm{NPF}_{6}$ as supporting electrolyte, $\mathrm{AgCl} / \mathrm{Ag}$ as reference electrode, gold disk as working electrode, $\mathrm{Au}$ as counter electrode, and scan rate at $50 \mathrm{mV} \mathrm{s}^{-1}$. The potential was externally calibrated against the ferrocene/ferrocenium couple. The single crystal was measured at low temperature ( $\mathrm{T}$ $=170 \mathrm{~K})$ on a Bruker D8 Discover equipped with a Pilatus $100 \mathrm{~K}$ large-area $2 \mathrm{D}$ detector and a $\mathrm{Cu}$ anode of wavelength $1.542 \AA$. The instrument was used with a parallel-beam geometry. 


\subsection{Synthetic procedures and characterization data}

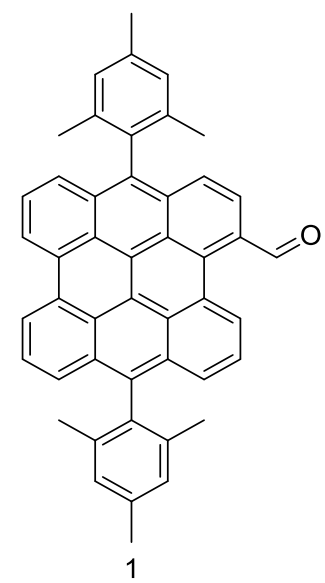

$\mathrm{POCl}_{3}(521.32 \mathrm{mg}, 3.4 \mathrm{mmol})$ was added dropwise to $3 \mathrm{~mL}$ dry $N, N$ Dimethylformamide (DMF), and the mixture was stirred at $0{ }^{\circ} \mathrm{C}$ under nitrogen atmosphere. The reaction mixture was allowed to warm to room temperature and stirred for $24 \mathrm{~h}$. Then the resulting mixture was added to the other two-neck round-bottom flask containing dry $3 \mathrm{~mL}$ dichloroethane (DCE) and compound Bisanthene-Mes ${ }^{\mathrm{S}}$ (100 mg). The reaction mixture was heated in oil bath at $90{ }^{\circ} \mathrm{C}$ for $3 \mathrm{~h}$ till it turned to purple. The solution was then cooled to room temperature, neutralized with $20 \mathrm{w} \%$ aqueous $\mathrm{NaOH}$, extracted by $\mathrm{CHCl}_{3}$, and dried over $\mathrm{Na}_{2} \mathrm{SO}_{4}$. Solvents were then removed under reduced pressure and the residue was purified by column chromatography ( dark green solid (69 mg, 69\% yield); ${ }^{1} \mathrm{H}$ NMR (500 MHz, $\left.\mathrm{CDCl}_{3}\right): \delta(\mathrm{ppm})=9.85(\mathrm{~s}$, $1 \mathrm{H}), 8.08(\mathrm{~d}, J=5 \mathrm{~Hz}, 1 \mathrm{H}), 7.85(\mathrm{~d}, J=5.0 \mathrm{~Hz}, 1 \mathrm{H}), 7.81-7.79(\mathrm{~m}, 2 \mathrm{H}), 7.68-7.67(\mathrm{~m}$, 2H), $7.65(\mathrm{t}, J=7.5 \mathrm{~Hz}, 2 \mathrm{H}), 7.47-7.41(\mathrm{~m}, 3 \mathrm{H}), 7.20(\mathrm{~d}, J=5.0 \mathrm{~Hz}, 4 \mathrm{H}), 2.52(\mathrm{~s}$, 6H),1.91-1.89 (m, 12H); ${ }^{13} \mathrm{C}$ NMR $\left(125 \mathrm{MHz}, \mathrm{CDCl}_{3}\right): \delta(\mathrm{ppm})=192.7,137.9,137.8$, $137.7,137.7,137.7,137.5,134.2,132.9,129.6,128.7,128.6,128.5,128.4,128.4,128.4$, $128.2,126.7,126.4,126.3,126.2,125.9,125.8,125.5,125.3,124.4,21.3,20.3,20.3$, 20.1, 19.9; MALDI-TOF $(\mathrm{M}+\mathrm{H})^{+}$calcd for $\mathrm{C}_{47} \mathrm{H}_{34} \mathrm{O}$ 615.2682; found: 615.2688 (error: $0.97 \mathrm{ppm})$.

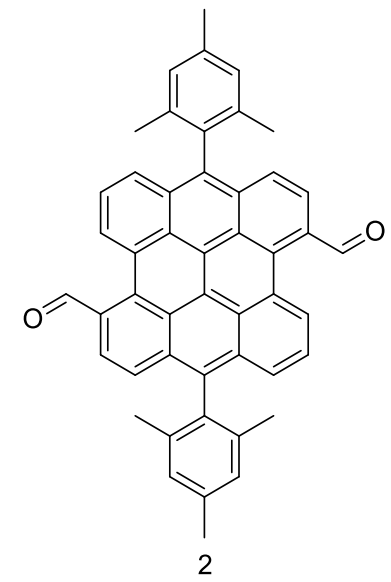

$\mathrm{POCl}_{3}(521.32 \mathrm{mg}, \quad 3.4 \mathrm{mmol})$ was added dropwise to $3 \mathrm{~mL}$ dry $N, N$ Dimethylformamide (DMF), and the mixture was stirred at $0{ }^{\circ} \mathrm{C}$ under nitrogen 
atmosphere. The reaction mixture was allowed to warm to room temperature and stirred for $24 \mathrm{~h}$. Then, the mixture was added to the other two-neck flask containing dry $3 \mathrm{~mL}$ dichloroethane (DCE) and compound Bisanthene-Mes $(100 \mathrm{mg})$. The reaction mixture was heated at $90^{\circ} \mathrm{C}$ for $5 \mathrm{~h}$ till it turned to purple. The solution was then cooled to room temperature, neutralized with $20 \mathrm{w} \%$ aqueous $\mathrm{NaOH}$, diluted in $\mathrm{CHCl}_{3}$ and dried over $\mathrm{Na}_{2} \mathrm{SO}_{4}$. Solvents were removed under reduced pressure and the residue was finally purified by column chromatography (silica gel, Hexane $/ \mathrm{DCM}=2: 1 \mathrm{v} / \mathrm{v}$ ) to give powder compound 2 as a dark green solid (52 mg, 52\% yield); ${ }^{1} \mathrm{H} \mathrm{NMR}\left(500 \mathrm{MHz}, \mathrm{CDCl}_{3}\right): \delta$ $(\mathrm{ppm})=9.78(\mathrm{~s}, 2 \mathrm{H}), 8.18(\mathrm{~d}, J=5.0 \mathrm{~Hz}, 2 \mathrm{H}), 7.88-7.83(\mathrm{~d}, 4 \mathrm{H}), 7.67(\mathrm{~d}, J=5 \mathrm{~Hz}, 2 \mathrm{H})$, 7.52-7.46 (d, 2H), $7.19(\mathrm{~s}, 4 \mathrm{H}), 2.52(\mathrm{~s}, 6 \mathrm{H}), 1.88(\mathrm{~s}, 12 \mathrm{H}) ;{ }^{13} \mathrm{C} \mathrm{NMR}(125 \mathrm{MHz}$, $\left.\mathrm{CDCl}_{3}\right): \delta(\mathrm{ppm})=192.7,138.9,138.2,138.1,138.0,137.6,137.5,136.9,136.8,135.6$, $135.3,135.1,133,5,133.5,133.0,132.3,131.9,131.8,131.4,130.3,129.6,129.0,128.9$, $128.8,128.7,128.7,128.6,127.7,127.5,126.6,126.1,126.0,125.9,125.8,125.6,125.3$, $125.2,124.8,124.8,123.5,21.3,20.3,20.2,20.1$; MALDI-TOF $(\mathrm{M}+\mathrm{H})^{+}$calcd for $\mathrm{C}_{48} \mathrm{H}_{34} \mathrm{O}_{2}$ 643.2632; found: 643.2629 (error: -0.47 ppm).

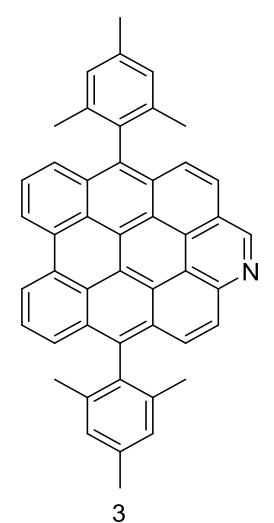

Trifluoroacetic acid (TFA) (1.0 mL) and Trifluoromethanesulfonic acid (TfOH) (150.08 $\mathrm{mg}, 1.0 \mathrm{mmol})$ were added sequentially into a $25 \mathrm{~mL}$ two-neck flask containing $\mathbf{1}$ (122.25 mg, $0.2 \mathrm{mmol}$ ) and azidotrimethylsilane (TMSN 3 ) (46.08 mg, $0.4 \mathrm{mmol}$ ) under air. The resulting solution was stirred in oil bath at $60^{\circ} \mathrm{C}$ for $8 \mathrm{~h}$. The reaction mixture was then diluted with ethyl acetate, washed with aqueous $\mathrm{NaOH}(2 \mathrm{M}, 30 \mathrm{~mL})$, and dried over anhydrous $\mathrm{Na}_{2} \mathrm{SO}_{4}$. Solvents were removed via rotary evaporator. The crude product was further purified by flash column chromatography (silica gel, ethyl acetate $/$ hexane $=1: 2 \mathrm{v} / \mathrm{v})$ to give powder compound 3 as a dark green solid $(99 \mathrm{mg}, 81 \%$ yield); ${ }^{1} \mathrm{H}$ NMR $\left(500 \mathrm{MHz}, \mathrm{CDCl}_{3}\right): \delta(\mathrm{ppm})=9.07(\mathrm{~d}, \mathrm{~J}=10.0 \mathrm{~Hz}, 1 \mathrm{H}), 7.75-7.67(\mathrm{~m}$, $1 \mathrm{H}), 7.64-7.63(\mathrm{~m}, 3 \mathrm{H}), 7.60-7.53(\mathrm{~m}, 2 \mathrm{H}), 7.42-7.38(\mathrm{~m}, 2 \mathrm{H}), 7.22-7.15(\mathrm{~m}, 4 \mathrm{H}), 6.95$ $(\mathrm{s}, 1 \mathrm{H}), 6.93(\mathrm{~m}, 1 \mathrm{H}), 2.52-2.04(\mathrm{~m}, 6 \mathrm{H}), 2.00-1.86(\mathrm{~m}, 12 \mathrm{H}) ;{ }^{13} \mathrm{C}$ NMR $(125 \mathrm{MHz}$, $\left.\mathrm{CDCl}_{3}\right): \delta(\mathrm{ppm})=148.0,145.0,138.4,138.4,138.3,138.3,138.2,134.9,134.5,130.4$, 129.7, 129.4, 129.1, 129.0, 128.9, 127.1, 127.1, 126.7, 125.4, 122.6, 119.2, 116.8, 21.6, 21.6, 20.7, 20.7, 20.6; MALDI-TOF $(\mathrm{M}+\mathrm{H})^{+}$calcd for $\mathrm{C}_{47} \mathrm{H}_{33} \mathrm{~N}$ 612.2686; found: 612.2693 (error: 1.14 ppm). 

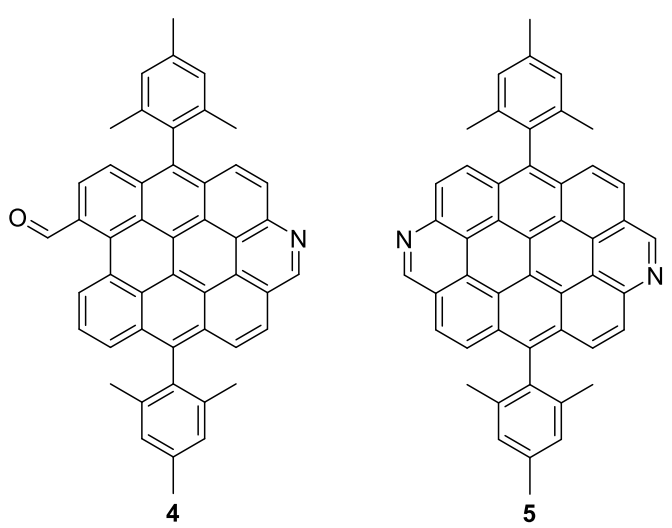

Table S1 Optimization of one-step synthesis of $\mathbf{4}$ and $\mathbf{5}^{a}$

\begin{tabular}{cccccc}
\hline Entry & $\begin{array}{c}\text { Amount } / \mathrm{mmoL} \\
(\mathrm{TfOH})\end{array}$ & $\begin{array}{c}\text { Temperature } \\
/{ }^{\circ} \mathrm{C}\end{array}$ & Time $/ \mathrm{h}$ & $\mathbf{4}$ Yield $/ \%^{b}$ & $\mathbf{5}$ Yield $/ \%^{b}$ \\
\hline 1 & 1 & 50 & 4 & 18 & 25 \\
2 & 1 & 60 & 4 & 22 & 29 \\
3 & 1 & 70 & 4 & 16 & 21 \\
4 & 1 & 80 & 4 & 11 & 15 \\
5 & 1 & 60 & 6 & 27 & 36 \\
6 & 1 & 60 & 8 & 32 & 41 \\
7 & 1 & 60 & 10 & 39 & 48 \\
8 & 1 & 60 & 12 & 44 & 53 \\
9 & 1 & 60 & 14 & 35 & 41 \\
10 & 0.5 & 60 & 12 & 22 & 37 \\
11 & 1.5 & 60 & 12 & 41 & 52 \\
\hline
\end{tabular}

${ }^{a}$ Reaction conditions: $2(0.2 \mathrm{mmol})$, azidotrimethylsilane $\left(\mathrm{TMSN}_{3}\right)$ (46.08 $\mathrm{mg}, 0.4$ mmol), Trifluoromethanesulfonic acid $(\mathrm{TfOH})(150.08 \mathrm{mg}, 1.0 \mathrm{mmol})$ and Trifluoroacetic acid (TFA) $(1.0 \mathrm{~mL})$ were added to a $25 \mathrm{~mL}$ two-neck flask under air. ${ }^{b}$ Isolated yield.

Taking entry 8 as an example to detailly explain the reaction procedure:

2 (127.25 mg, $0.2 \mathrm{mmol})$ and azidotrimethylsilane $\left(\mathrm{TMSN}_{3}\right)$ (46.08 mg, $\left.0.4 \mathrm{mmol}\right)$ were added to a $25 \mathrm{~mL}$ two-neck flask under air, then trifluoroacetic acid (TFA) (1.0 $\mathrm{mL})$ and trifluoromethanesulfonic acid $(\mathrm{TfOH})(150.08 \mathrm{mg}, 1.0 \mathrm{mmol})$ were added to the mixture sequentially. The resulting mixture was stirred in oil bath at $60{ }^{\circ} \mathrm{C}$ for $12 \mathrm{~h}$. Then the reaction mixture was cooled down, diluted with ethyl acetate, washed with aqueous $\mathrm{NaOH}(2 \mathrm{M}, 30 \mathrm{~mL})$ and dried over anhydrous $\mathrm{Na}_{2} \mathrm{SO}_{4}$. Solvents were removed via rotary evaporator and the crude product was further purified by flash column chromatography (silica gel, ethyl acetate/hexane $=1: 2 \mathrm{v} / \mathrm{v}$ ) to give powder compound 4 as a purple black solid (55.99 mg, 44\% yield) and powder compound $\mathbf{5}$ as a yellow solid (67.44 mg, 53\% yield); Compound 4: ${ }^{1} \mathrm{H}$ NMR (500 MHz, $\left.\mathrm{CDCl}_{3}\right)$ : $\delta$ $(\mathrm{ppm})=10.41(\mathrm{~d}, J=10.0 \mathrm{~Hz}, 1 \mathrm{H}), 9.92(\mathrm{~s}, 1 \mathrm{H}), 8.45(\mathrm{~d}, J=10.0 \mathrm{~Hz}, 1 \mathrm{H}), 8.33(\mathrm{~d}, J=$ $5.0 \mathrm{~Hz}, 1 \mathrm{H}), 8.29-8.21(\mathrm{~m}, 4 \mathrm{H}), 8.12(\mathrm{~d}, J=10.0 \mathrm{~Hz}, 1 \mathrm{H}), 8.03(\mathrm{~d}, J=10.0 \mathrm{~Hz}, 1 \mathrm{H})$, 7.84(d, $J=10.0 \mathrm{~Hz}, 1 \mathrm{H}), 7.27(\mathrm{~s}, 4 \mathrm{H}), 2.58(\mathrm{~s}, 6 \mathrm{H}), 1.91(\mathrm{~s}, 12 \mathrm{H})$; Compound $4:{ }^{13} \mathrm{C}$ NMR $\left(125 \mathrm{MHz}, \mathrm{CDCl}_{3}\right): \delta(\mathrm{ppm})=192.9,138.1,138.0,137.9,137.9,137.9,137.7$, 
134.4, 133.1, 129.6, 128.9, 128.8, 128.7, 128.7, 128.6, 128.4, 126.9, 126.6, 126.5, 126.4,

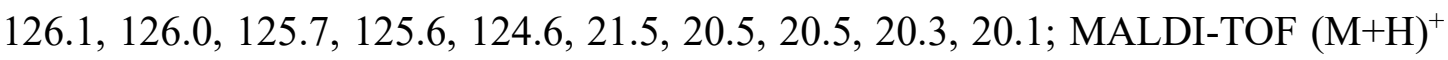
calcd for $\mathrm{C}_{48} \mathrm{H}_{33} \mathrm{NO}$ 640.2635; found: 640.2641 (error: 0.94 ppm). Compound 5: ${ }^{1} \mathrm{H}$ NMR (500 MHz, $\left.\mathrm{CDCl}_{3}\right): \delta(\mathrm{ppm}) 10.69(\mathrm{~s}, 2 \mathrm{H}), 9.32(\mathrm{~d}, J=10.0 \mathrm{~Hz}, 2 \mathrm{H}), 9.13(\mathrm{~d}, J$ $=5.0 \mathrm{~Hz}, 2 \mathrm{H}), 9.06(\mathrm{~d}, J=5.0 \mathrm{~Hz}, 2 \mathrm{H}), 8.83(\mathrm{~d}, J=10.0 \mathrm{~Hz}, 2 \mathrm{H}), 7.41(\mathrm{~s}, 4 \mathrm{H}), 2.68(\mathrm{~s}$, 6H), 1.92 (s, 12H); Compound 5: ${ }^{13} \mathrm{C}$ NMR (125 MHz, $\left.\mathrm{CDCl}_{3}\right): \delta(\mathrm{ppm}) 147.9,144.9$, 138.3, 138.3 , 138.2, 138.1, 138.1, 134.8, 134.4, 130.3, 129.6, 129.2, 128.9, 128.9, 128.8, 127.0, 126.9, 126.6, 125.3, 122.5, 121.9, 119.9, 119.1, 116.3, 21.5, 20.6, 20.5, 20.5;

MALDI-TOF $(\mathrm{M}+\mathrm{H})^{+}$calcd for $\mathrm{C}_{48} \mathrm{H}_{32} \mathrm{~N}_{2}$ 637.2638; found: 637.2627 (error: -1.73 ppm).

\subsection{Gram Scale}

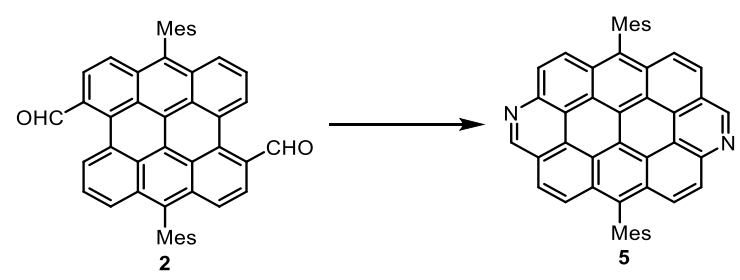

2 (642.26 mg, $1 \mathrm{mmol}), \mathrm{TMSN}_{3}(2.304 \mathrm{~g}, 2 \mathrm{mmol})$ were added into a $150 \mathrm{~mL}$ two-neck flask containing $15 \mathrm{~mL}$ TFA under air, then TfOH (750.40 mg, $5.0 \mathrm{mmol}$ ) was added afterwards. The resulting mixture was stirred in oil bath at $60{ }^{\circ} \mathrm{C}$ for 12 hours. The mixture was then diluted with ethyl acetate, washed with aqueous $\mathrm{NaOH}(2 \mathrm{M}, 80 \mathrm{~mL})$, dried over anhydrous $\mathrm{Na}_{2} \mathrm{SO}_{4}$. Solvents were removed via rotary evaporator, and the crude product was further purified by flash column chromatography (silica gel, ethyl acetate/hexane $=1: 2 \mathrm{v} / \mathrm{v})$ to give pure compound 5 as a yellow solid $(301.86 \mathrm{mg}, 47 \%$ yield). 


\section{Acid response}

The procedure for titrating 5 with $\mathrm{HCl}$ is:

1) Mix $12 \mathrm{M} \mathrm{HCl}$ and 2e-5 M 5 (in $\mathrm{THF}$ ) in 1:1 volume ratio to generate $6 \mathrm{M} \mathrm{HCl}$ stock solution $\mathbf{A}$.

2) Prepare 1e-5 M 5 stock solution $\mathbf{B}$.

3) Tune the volume ratio of $\mathbf{A}$ and $\mathbf{B}$ to generate different concentrations of $\mathrm{HCl}$, and measure after each mixing.

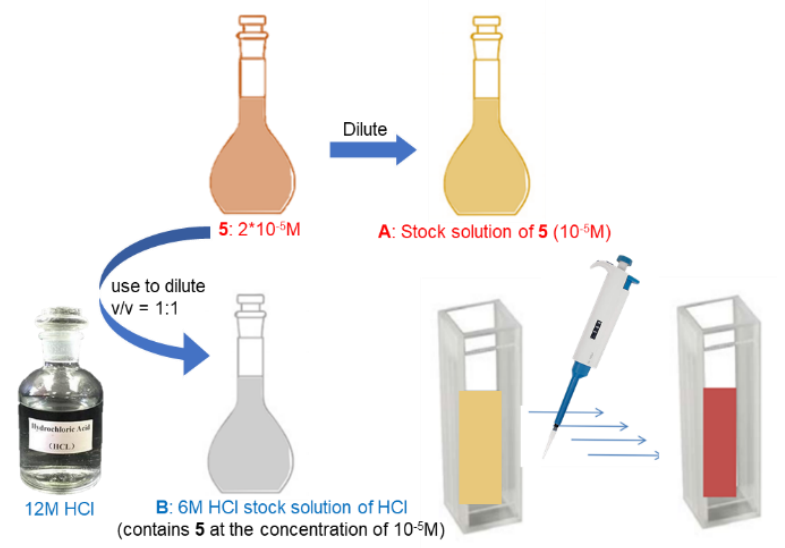

Figure S1. Titration process of 5 by $\mathrm{HCl}$.
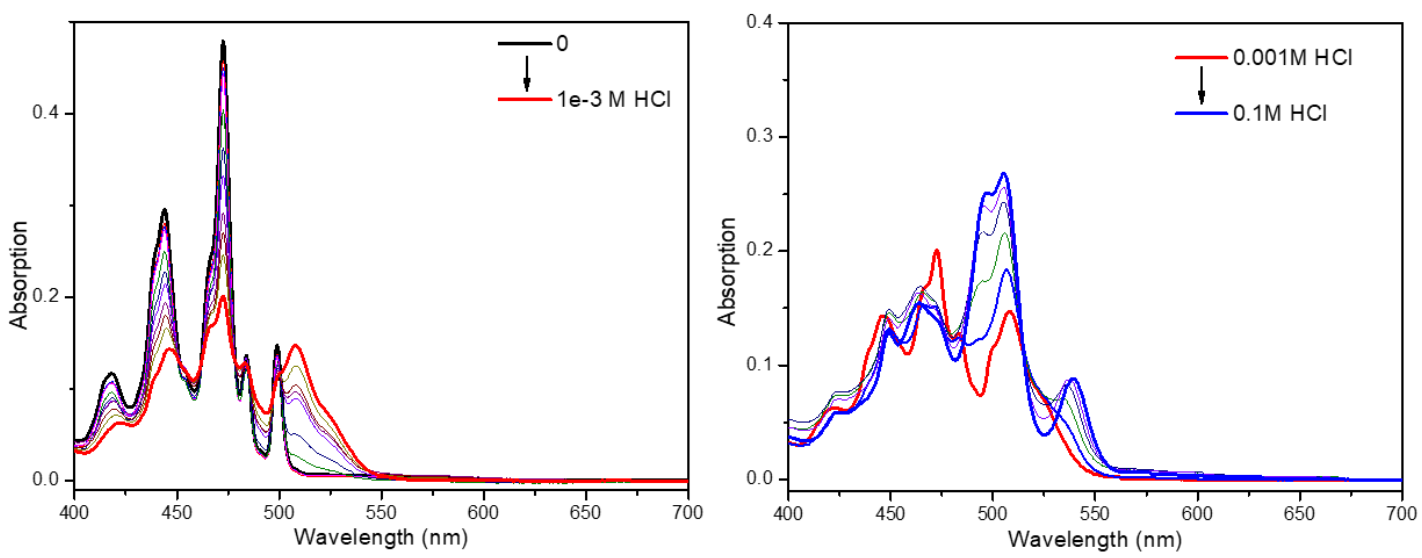

Figure S2. Change of the absorption spectrum of $\mathbf{5}$ upon titration with $\mathrm{HCl}$ in THF. 
Titration curve-fitting and association constant values were calculated by employing the BindFit program developed by Prof. Pall Thordarson of UNSW. ${ }^{\text {S1 }}$ 1:2 Binding stoichiometry was chosen in the BindFit program. This program employs a nonlinear least-squares regression analysis and is available free of cost online through the following link: http://supramolecular.org.

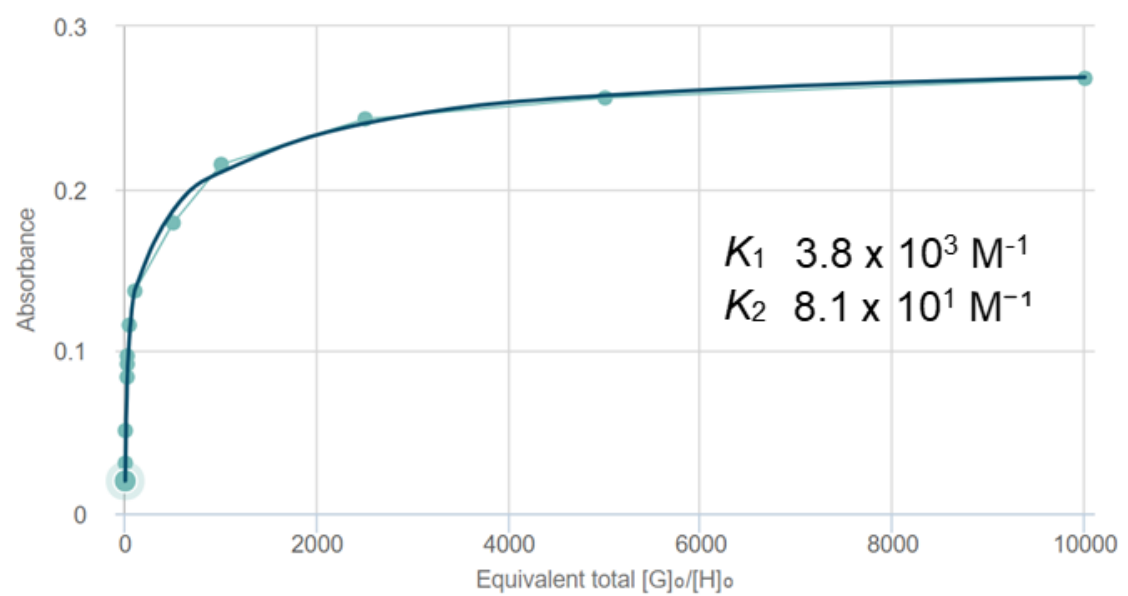

Figure S3. Plot of the absorbance at $505 \mathrm{~nm}$ with a fitting curve (titration by $\mathrm{HCl}$ in THF at $298 \mathrm{~K}$ ).

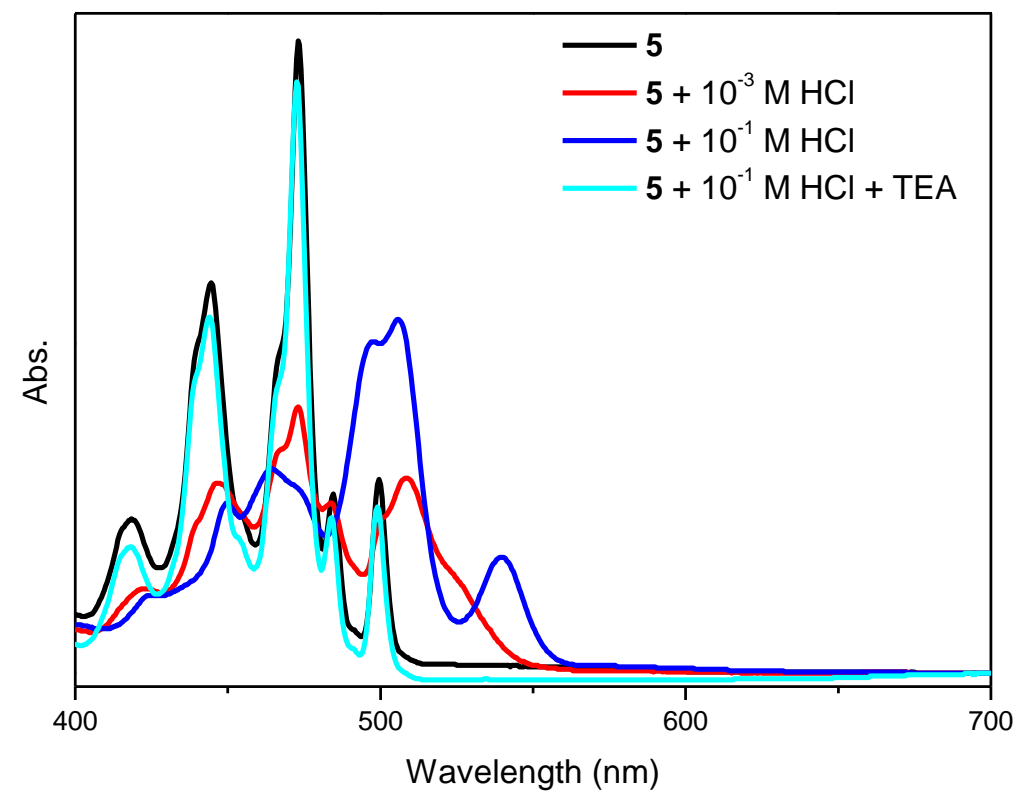

Figure S4. Acid ( $\mathrm{HCl})$-induced UV-Vis absorption changes of compound $\mathbf{5}$ in THF, and finally neutralized by TEA (Concentration of $\mathbf{5}$ is $10^{-5} \mathrm{M}$ ). 
The procedure for titrating $\mathbf{5}$ with TFA is similar as $\mathrm{HCl}$ :
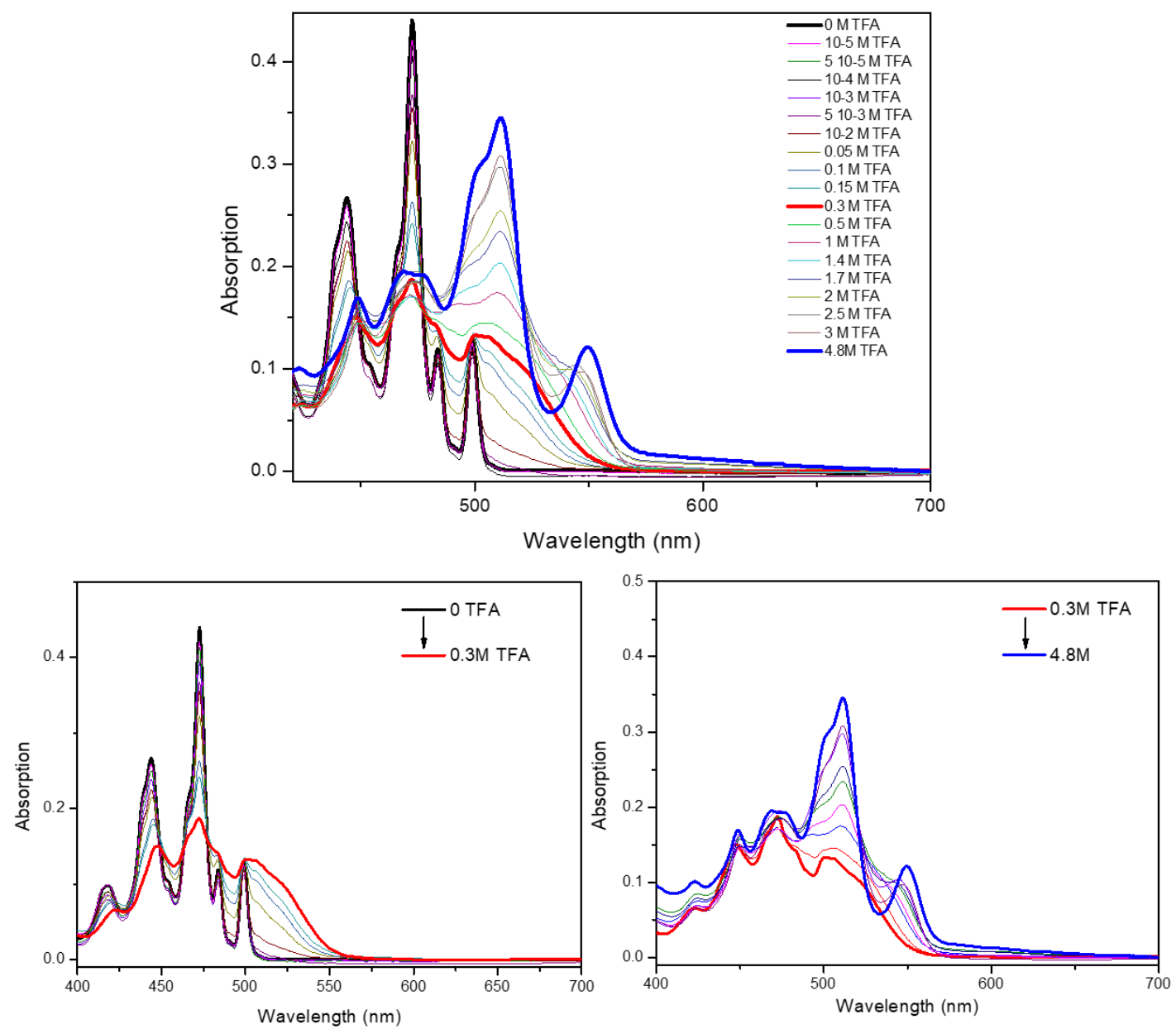

Figure S5. Change of the absorption spectrum of $\mathbf{5}$ upon titration by TFA in THF. 


\section{Cyclic voltammetry}

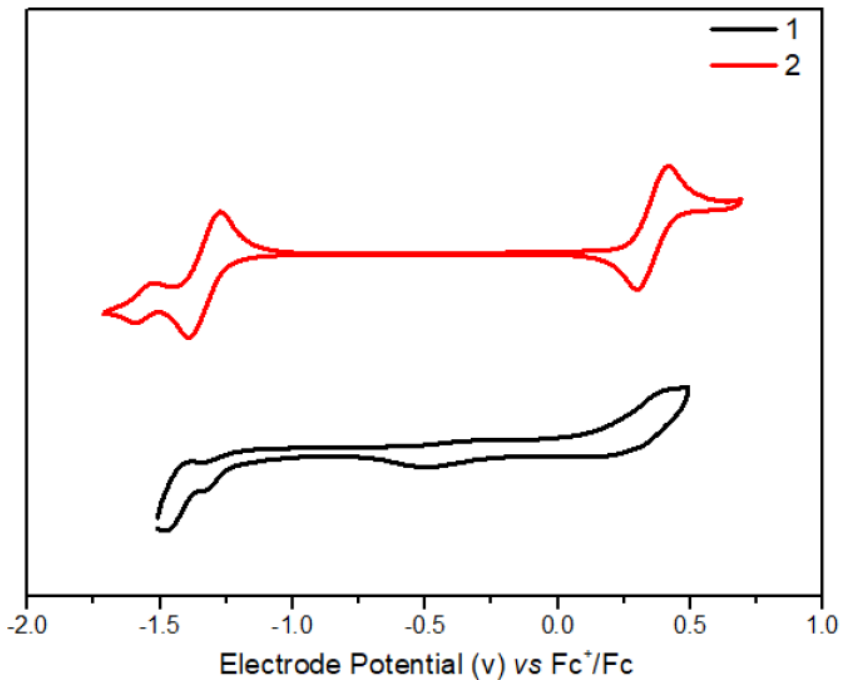

Figure S6. Cyclic voltammogram of $\mathbf{1 , 2}$ in $\mathrm{DCM}$ with $0.1 \mathrm{M} \mathrm{Bu}_{4} \mathrm{NPF}_{6}$ as a supporting electrolyte. 


\section{DFT calculations}

(a)

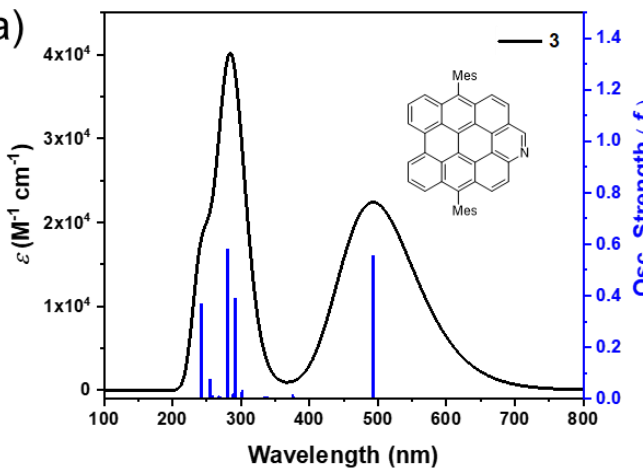

(c)

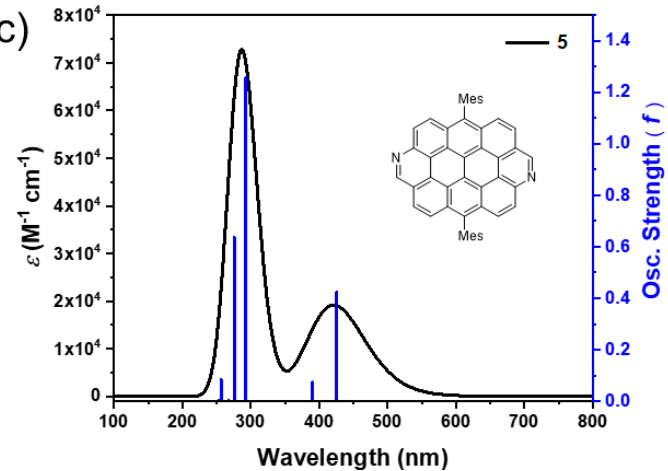

(b)

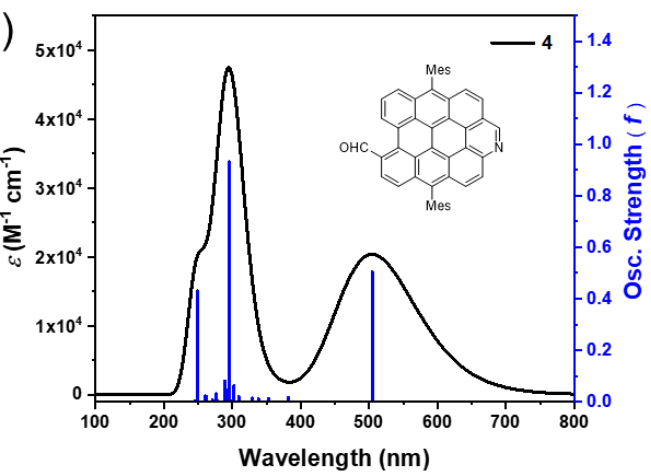

(d)

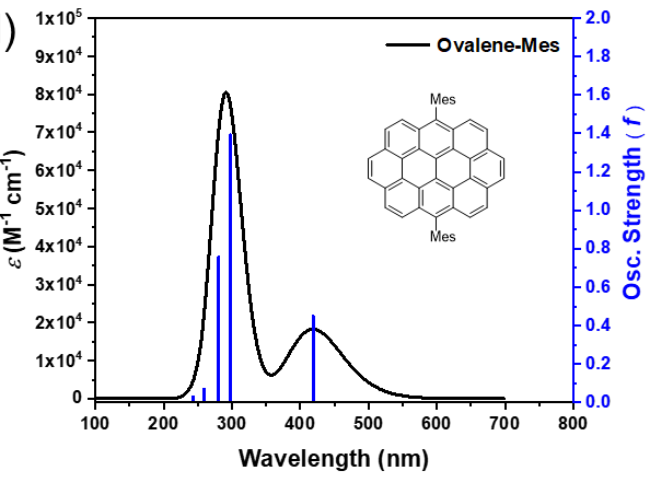

Figure S7. TD-DFT (CAM-B3LYP/6-31G*) simulated UV-Vis absorption spectra with oscillator strength of compound (a) 3, (b) 4, (c) 5 and (d) Ovalene-Mes.

Table S2. Calculated (B3LYP/6-31G*) HOMO and LUMO energy diagrams of $\mathbf{3}, \mathbf{4}, \mathbf{5}$ and Ovalene-Mes.

\begin{tabular}{ccccc}
\hline compound & $\mathbf{3}$ & $\mathbf{4}$ & $\mathbf{5}$ & Ovalene-Mes \\
$\mathrm{LUMO} / \mathrm{eV}$ & -2.40 & -2.74 & -2.34 & -1.99 \\
$\mathrm{HOMO} / \mathrm{eV}$ & -4.78 & -5.06 & -5.18 & -4.85 \\
\hline
\end{tabular}

Table S3. Selected TD-DFT (CAM-B3LYP/6-31G*) calculated energies, oscillator strength and compositions of major electronic transitions of $\mathbf{3}, \mathbf{4}$ and 5. ${ }^{[3]}$

\begin{tabular}{|c|c|c|c|}
\hline Compound & $\begin{array}{c}\text { Wavelength } \\
(\mathbf{n m})\end{array}$ & $\begin{array}{c}\text { Osc. Strength } \\
(f)\end{array}$ & Major contributions \\
\hline \multirow{4}{*}{3} & 493.00 & 0.5530 & H -> L (97.8\%); \\
\hline & 291.39 & 0.3863 & H-1 -> L (27.8\%), H-7 -> L (20.7\%), \\
\hline & & & $\begin{array}{l}\mathrm{H}->\mathrm{L}+4(17.0 \%), \mathrm{H}->\mathrm{L}+1(13.8 \%), \\
\mathrm{H}-8 \text {-> L }(6.0 \%), \mathrm{H}->\mathrm{L}+2(5.8 \%) ;\end{array}$ \\
\hline & 281.06 & 0.5787 & $\begin{array}{l}\mathrm{H}->\mathrm{L}+4(28.2 \%), \mathrm{H}->\mathrm{L}+1(18.4 \%), \\
\mathrm{H}-2 \text {-> L }(13.8 \%), \mathrm{H}-1 \text {-> L }(13.4 \%), \\
\mathrm{H}-8 \text {-> L }(9.1 \%) ;\end{array}$ \\
\hline
\end{tabular}




\begin{tabular}{|c|c|c|c|}
\hline & 254.62 & 0.0707 & $\begin{array}{l}\text { H-1 -> L+1 (28.2\%), H-2 -> L+1 (17.8\%), } \\
\text { H-1 -> L+2 (11.8\%), H -> L+10 (8.8\%), } \\
\text { H-11 -> L (7.0\%), H-2 -> L+2 (5.5\%); } \\
\text { H-1 -> L+1 (48.0\%), H-2 -> L+1 (26.6\%), } \\
\text { H-1 -> L+2 (5.3\%), H-10 -> L (5.1\%); }\end{array}$ \\
\hline \multirow{5}{*}{4} & 504.47 & 0.5028 & H -> L (97.5\%); \\
\hline & 302.33 & 0.0618 & $\begin{array}{l}\text { H-7 -> L }(37.9 \%), \text { H -> L+3 (18.4\%), } \\
\text { H -> L+2 (9.1\%), H -> L+1 (8.5\%); }\end{array}$ \\
\hline & 295.71 & 0.9318 & $\begin{array}{l}\mathrm{H}-1 \text {-> L }(45.3 \%), \mathrm{H}->\mathrm{L}+1(27.3 \%) \\
\mathrm{H}->\mathrm{L}+4(5.3 \%), \mathrm{H}-4 \text {-> L }(5.3 \%)\end{array}$ \\
\hline & 288.99 & 0.0791 & $\begin{array}{l}\mathrm{H}->\mathrm{L}+4(59.6 \%), \mathrm{H}->\mathrm{L}+3(7.9 \%) \\
\mathrm{H}->\mathrm{L}+2(6.4 \%)\end{array}$ \\
\hline & 248.42 & 0.4301 & H-1 -> L+1 (74.9\%); \\
\hline \multirow{5}{*}{5} & 425.27 & 0.4227 & H -> L (87.4\%); \\
\hline & 390.11 & 0.0731 & $\begin{array}{l}\mathrm{H}->\mathrm{L}+1(55.2 \%), \mathrm{H}-1 \text {-> L }(35.1 \%) \\
\mathrm{H}->\mathrm{L}(6.3 \%)\end{array}$ \\
\hline & 292.85 & 1.2569 & H-1 -> L (58.6\%), H -> L+1 (38.2\%); \\
\hline & 275.96 & 0.6361 & H-1 -> L+1 (77.5\%), H-4 -> L (8.1\%); \\
\hline & 256.54 & 0.0818 & $\begin{array}{l}\mathrm{H}->\mathrm{L}+6(59.0 \%), \mathrm{H}-11 \text {-> L (21.1\%), } \\
\mathrm{H}-2 \text {-> L+2 (9.0\%); }\end{array}$ \\
\hline
\end{tabular}

(Assignment; $\mathrm{H}=\mathrm{HOMO}, \mathrm{L}=\mathrm{LUMO}, \mathrm{H}-1=\mathrm{HOMO}-1, \mathrm{~L}+1=\mathrm{LUMO}+1$, etc.)

(a)

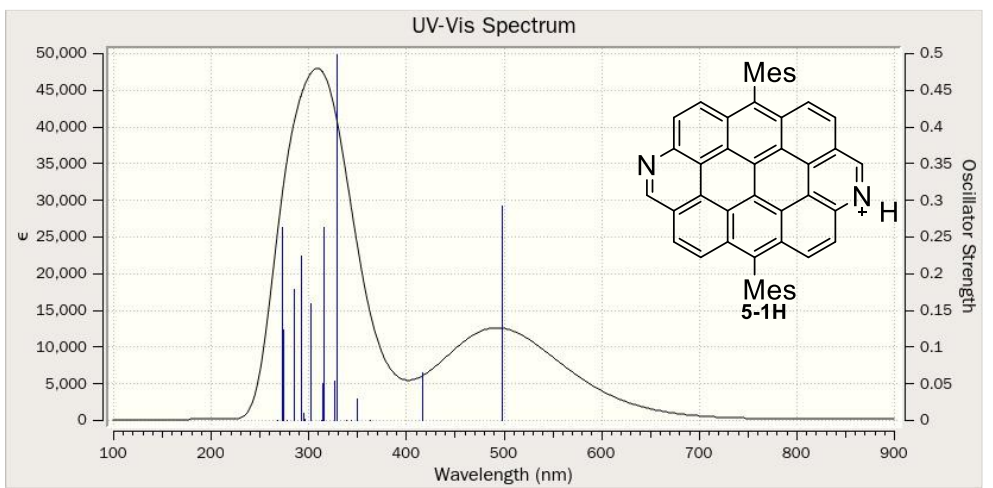

(b)

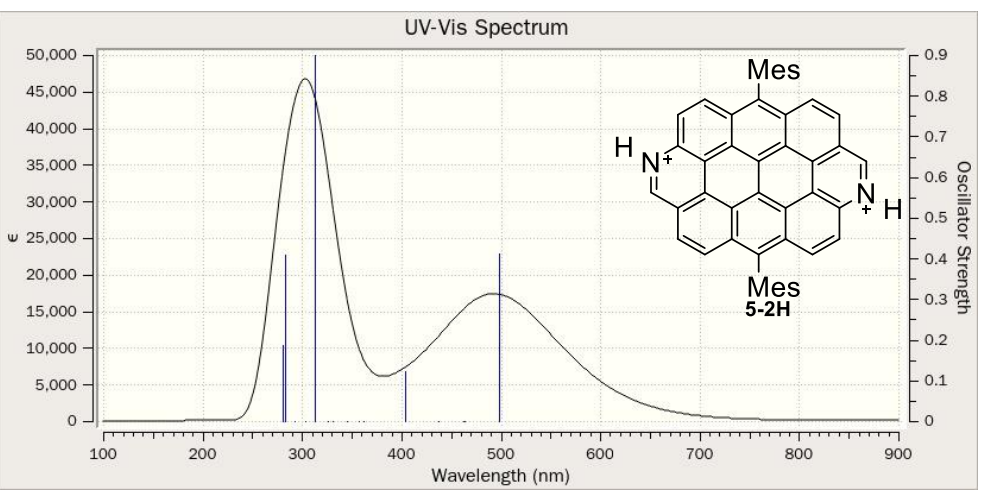

Figure S8. TD-DFT (CAM-B3LYP/6-31G*) simulated UV-Vis absorption spectra with oscillator strength of compound (a) 5-1H and (b) 5-2H. 
Table S4. Selected TD-DFT (CAM-B3LYP/6-31G*) calculated energies, oscillator strength and compositions of major electronic transitions of 5-H and 5-2H.

\begin{tabular}{|c|c|c|c|}
\hline Compound & $\begin{array}{c}\text { Wavelength } \\
(\mathbf{n m})\end{array}$ & $\begin{array}{c}\text { Osc. Strength } \\
(f)\end{array}$ & Major contributions \\
\hline \multirow[t]{9}{*}{$5-1 H$} & 498.51 & 0.2926 & H -> L (93.1\%); \\
\hline & 416.10 & 0.0655 & H -> L+1 (65.2\%), H-5 -> L (28.9\%); \\
\hline & 328.49 & 0.4990 & $\begin{array}{l}\mathrm{H}-5 \text {-> L }(38.8 \%), \mathrm{H}->\mathrm{L}+1(16.9 \%), \\
\mathrm{H}-2 \text {-> L }(15.2 \%), \mathrm{H}->\mathrm{L}+2(14.0 \%) ;\end{array}$ \\
\hline & 315.31 & 0.2633 & $\begin{array}{l}\text { H -> L+2 (22.4\%), H-5 -> L+1 (20.6\%), } \\
\text { H-7 -> L (12.0\%), H -> L+3 (9.9\%), } \\
\text { H -> L+1 (8.7\%), H-5 -> L (8.0\%), } \\
\text { H-6 -> L (5.8\%); }\end{array}$ \\
\hline & 302.17 & 0.1599 & $\begin{array}{l}\text { H -> L+2 (26.9\%), H-6 -> L+1 (22.4\%), } \\
\text { H-7 -> L (11.6\%), H-7 -> L+1 (10.3\%), } \\
\text { H-5 -> L+1 (10.1\%); }\end{array}$ \\
\hline & 292.42 & 0.2239 & $\begin{array}{l}\text { H -> L+3 }(49.3 \%), H-6 \text {-> L+1 (14.5\%), } \\
\text { H-5 -> L (6.9\%), H -> L+2 (6.0\%); }\end{array}$ \\
\hline & 285.45 & 0.1785 & $\begin{array}{l}\text { H-6 -> L+1 (37.2\%), H-5 -> L+1 (12.8\%), } \\
\text { H-7 -> L+1 (8.0\%), H-9 -> L (7.9\%), } \\
\text { H-5 -> L+2 (6.0\%); }\end{array}$ \\
\hline & 274.71 & 0.1232 & $\begin{array}{l}\text { H-9 -> L (40.7\%), H-10 -> L (15.8\%), } \\
\text { H -> L+2 (7.2\%), H -> L+3 (5.5\%); }\end{array}$ \\
\hline & 272.79 & 0.2625 & $\begin{array}{l}\mathrm{H}->\mathrm{L}+4(28.2 \%), \mathrm{H}-7 \text {-> L+1 }(26.1 \%) \\
\mathrm{H}->\mathrm{L}+2(8.6 \%), \mathrm{H}-9 \text {-> L }(8.2 \%) \\
\text { H-5 -> L+1 }(8.0 \%), \mathrm{H}-10 \text {-> L }(6.1 \%)\end{array}$ \\
\hline \multirow[t]{5}{*}{$5-2 H$} & 497.79 & 0.4106 & H -> L (93.8\%); \\
\hline & 404.25 & 0.1227 & H -> L +1 (70.1\%), H-5 -> L (26.8\%); \\
\hline & 312.78 & 0.8996 & H-5 -> L (64.7\%), H -> L+1 (25.0\%); \\
\hline & 283.41 & 0.4098 & H-5 -> L+1 (77.0\%), H-8 -> L (7.6\%); \\
\hline & 280.01 & 0.1870 & $\begin{array}{l}\text { H-8 -> L (57.3\%), H-6 -> L+2 (19.1\%), } \\
\text { H-5 -> L+1 (5.8\%), H-13 -> L }(5.5 \%)\end{array}$ \\
\hline
\end{tabular}



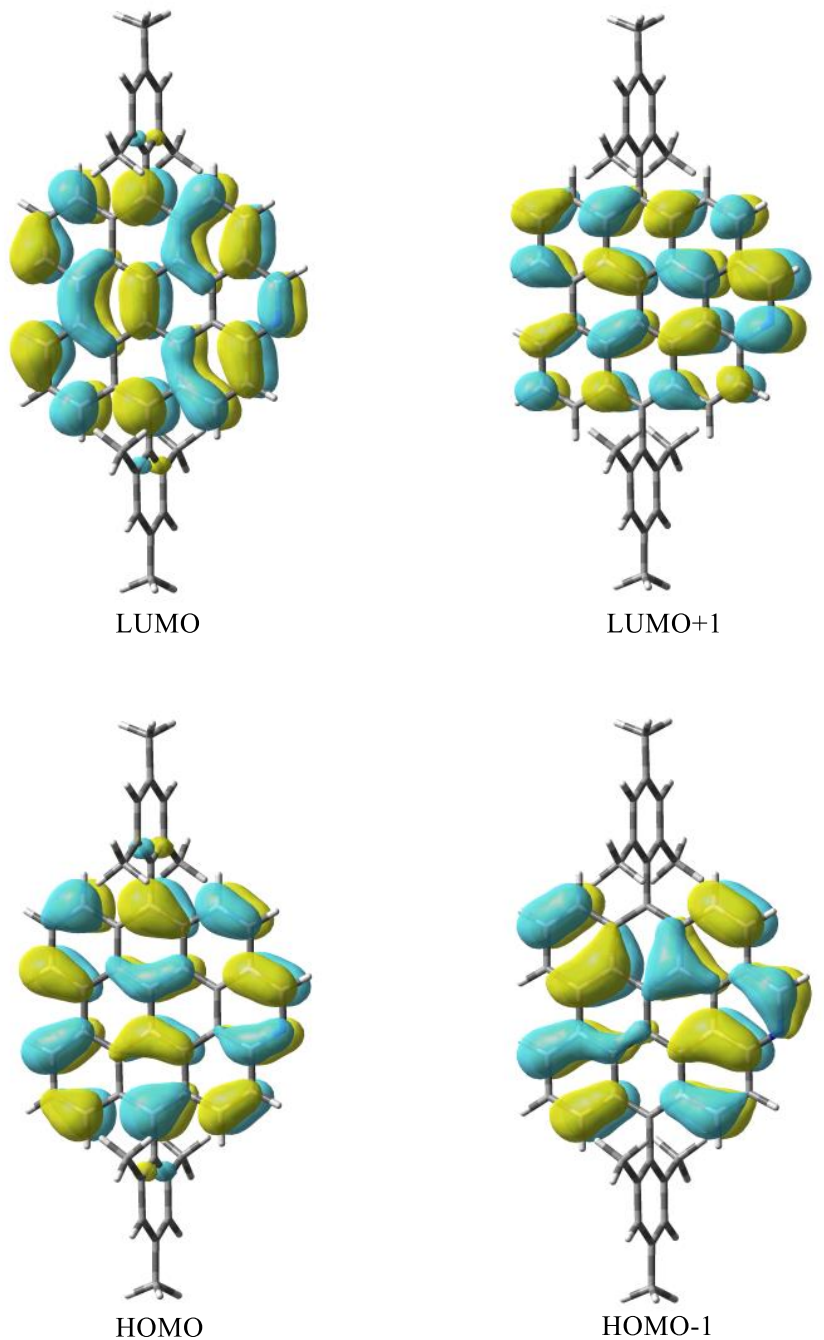

Figure S9. Calculated (B3LYP/6-31G*) LUMO, LUMO+1, HOMO and HOMO-1 of 3. 


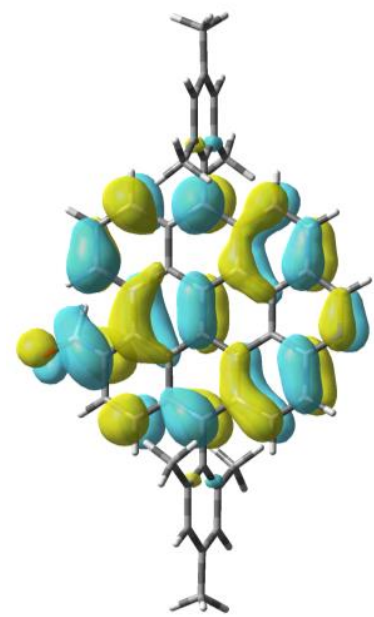

LUMO

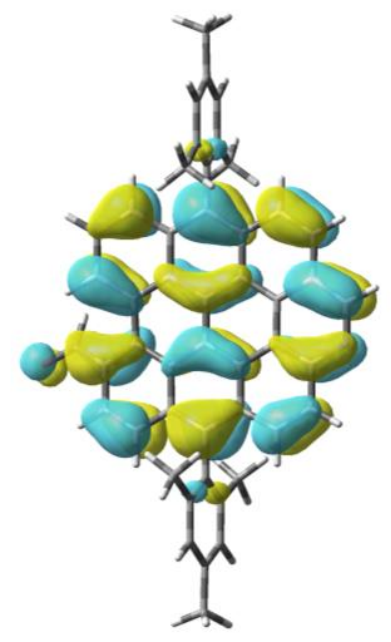

HOMO

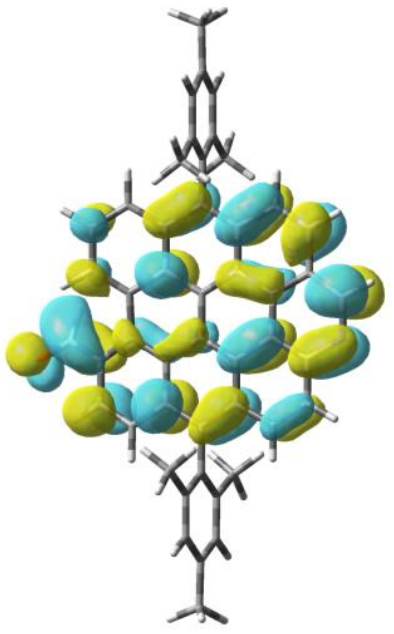

LUMO+1

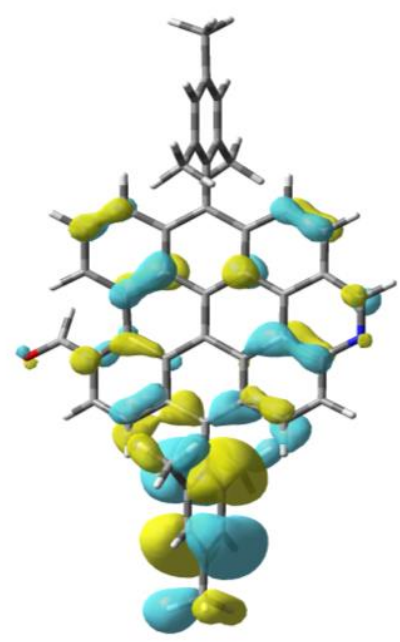

HOMO-1

Figure S10. Calculated (B3LYP/6-31G*) LUMO, LUMO+1, HOMO and HOMO-1 of 4 . 


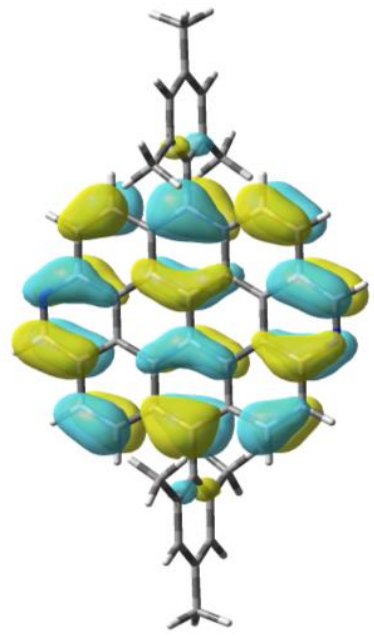

LUMO

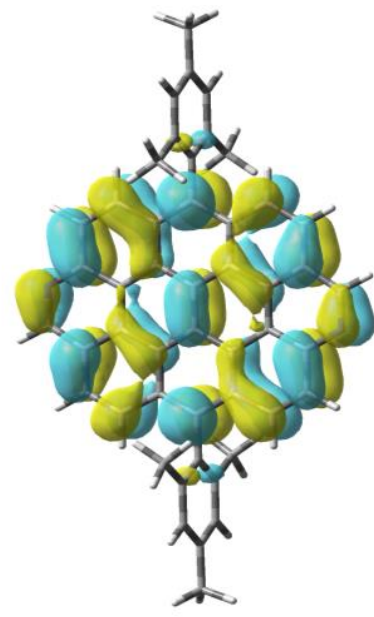

HOMO

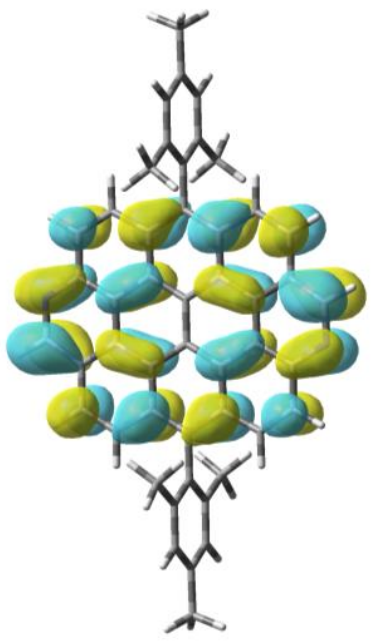

LUMO+1

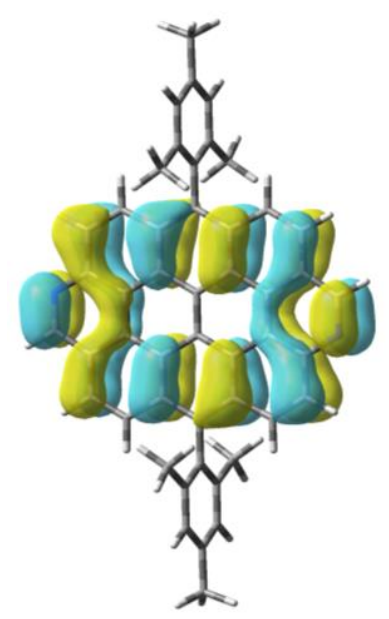

HOMO-1

Figure S11. Calculated (B3LYP/6-31G*) LUMO, LUMO+1, HOMO and HOMO-1 of 5. 


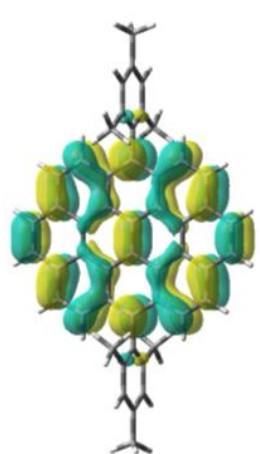

LUMO

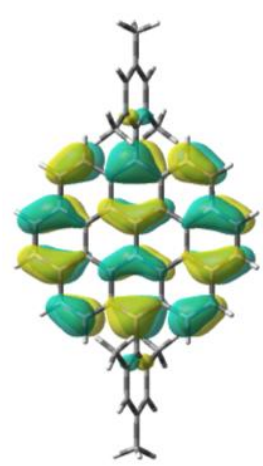

$\mathrm{HOMO}$

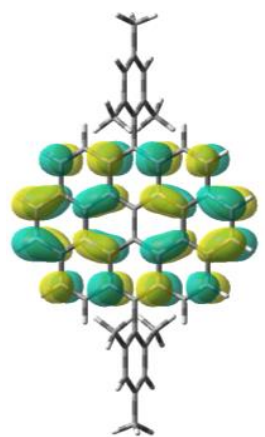

LUMO+1

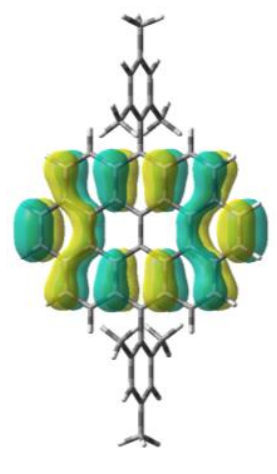

HOMO-1

Figure S12. Calculated (B3LYP/6-31G*) LUMO, LUMO+1, HOMO and HOMO-1 of Ovalene-Mes.
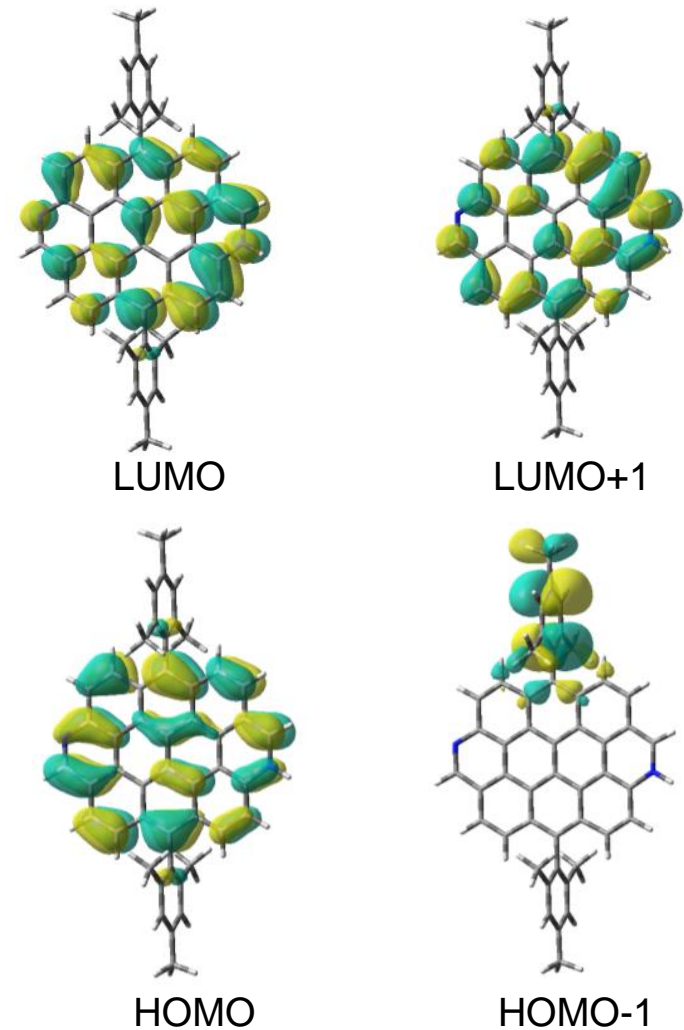

$\mathrm{HOMO}$

HOMO-1

Figure S13. Calculated (B3LYP/6-31G*) LUMO, LUMO+1, HOMO and HOMO-1 of 5-1H. 

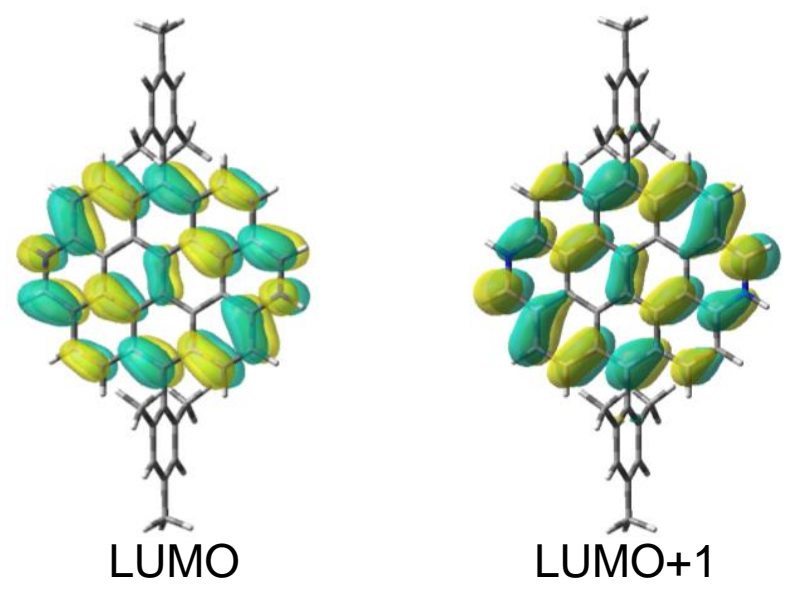

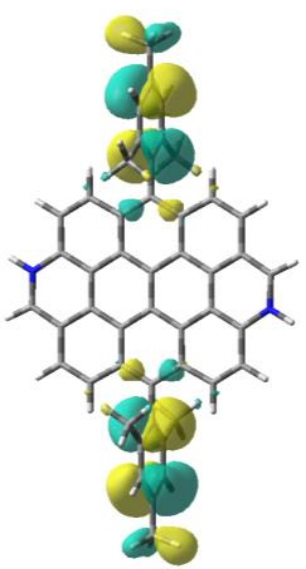

HOMO

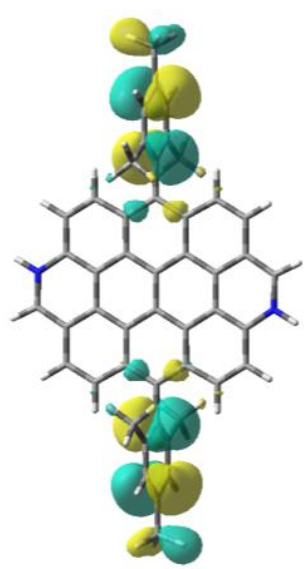

HOMO-1

Figure S14. Calculated (B3LYP/6-31G*) LUMO, LUMO+1, HOMO and HOMO-1 of 5-2H. 

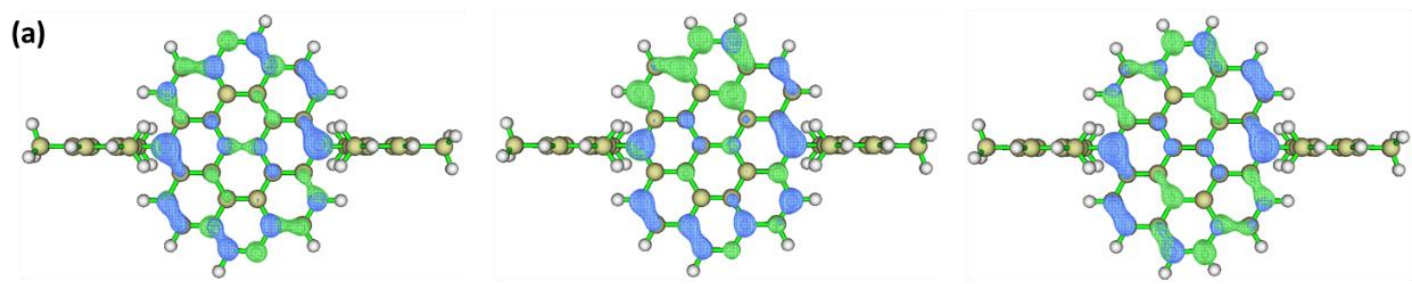

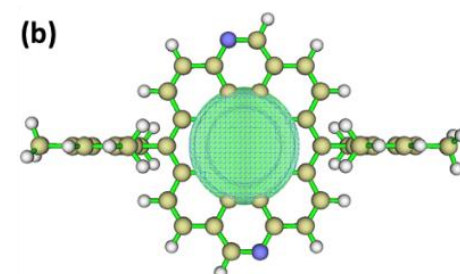

5

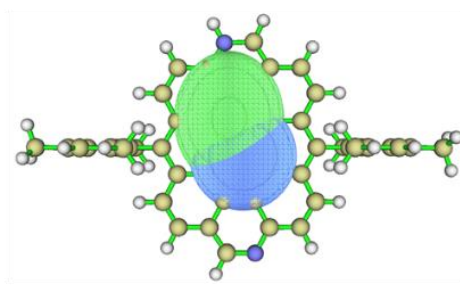

$5-\mathrm{H}$

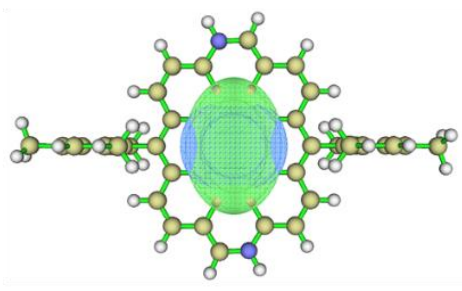

$5-2 \mathrm{H}$

Figure S15. (a) the hole and electron distribution for excited state $\mathrm{S} 0 \rightarrow \mathrm{S} 1$, the blue mesh surface stand for hole distribution, green color for electron distribution (isovalue $=0.002$ ); (b) Cele and Chole functions for excited state $\mathrm{S} 0 \rightarrow \mathrm{S} 1$, smoothing graphical description of hole and electron distribution which the center of blue (hole) and green (electron) elliptical iso-surfaces corresponding to the centroid positions of holes and electrons, respectively. (isovalue $=0.001)^{\mathrm{S} 4}$

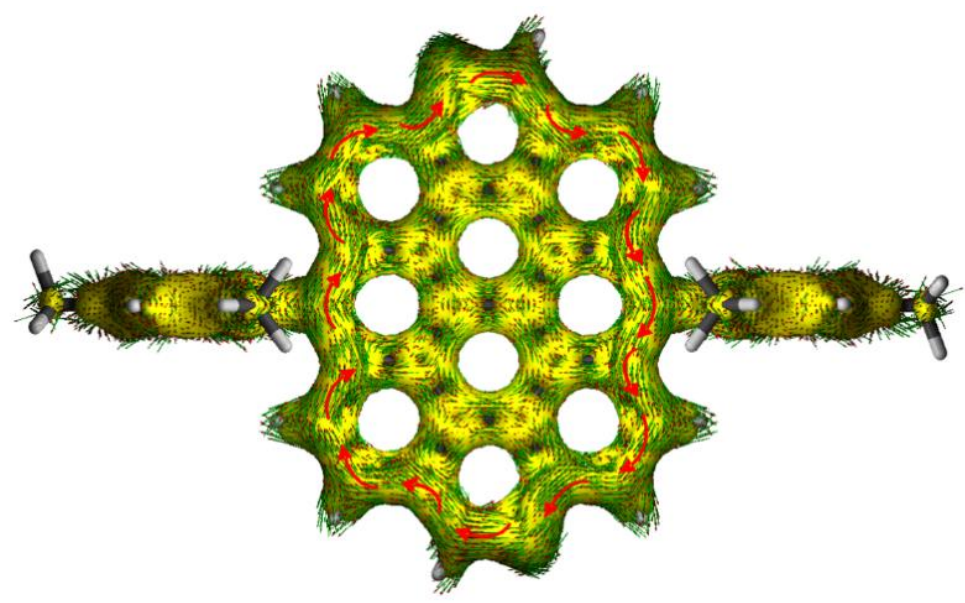

Figure S16. Calculated AICD plots including $\pi$ and $\sigma$ electrons contribution of 5 (isovalue $=0.05)$. The red circles labeled by arrows reflect the global ring current flow direction. 


\section{Appendix I: X-ray crystallographic data}

Single crystals of $\mathbf{5}$ suitable for X-ray crystallographic analysis were obtained by slow evaporation of $\mathbf{5}$ in dichloromethane and methanol solution.

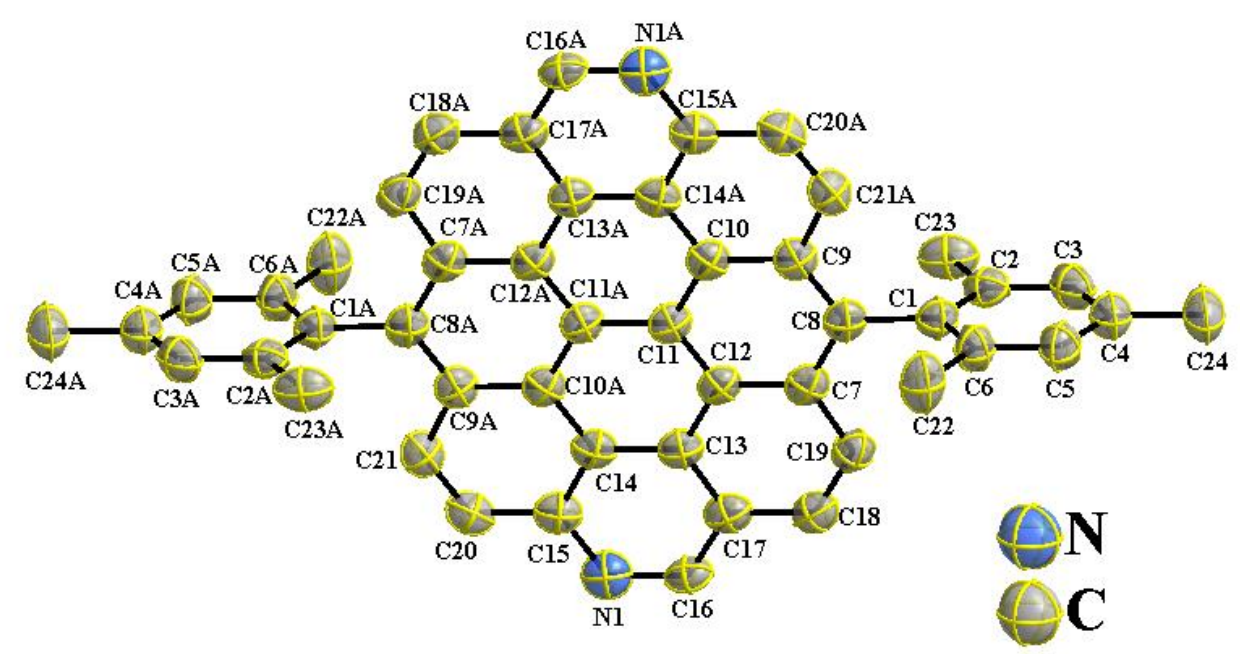

Figure S17. ORTEP diagrams for the molecular structure of 5. Thermal ellipsoids are drawn at the $50 \%$ probability level. Hydrogen atoms have been omitted for the sake of clarity. 
Table S5. Crystallographic data for 5 .

$\begin{array}{lll}\text { Empirical formula } & \mathrm{C}_{48} \mathrm{H}_{32} \mathrm{~N}_{2} & 636.75 \\ \text { Formula weight } & 170.00(10) \mathrm{K} & \\ \text { Temperature } & 1.54184 \AA & \\ \text { Wavelength } & \text { monoclinic } & \alpha=90^{\circ} \\ \text { Crystal system } & \mathrm{P} 21 / \mathrm{c} & \beta=99.820(2)^{\circ} \\ \text { Space group } & \mathrm{a}=8.4689(2) \AA & \gamma=90^{\circ} \\ \text { Unit cell dimensions } & \mathrm{b}=24.9000(4) \AA & \\ & \mathrm{c}=7.64750(10) \AA & \\ & 1589.04(5) \AA^{3} & \\ \text { Volume } & 2 & 1.331 \mathrm{~g} / \mathrm{cm}^{3} \\ \text { Z } & 0.589 \mathrm{~mm}^{-1} & 668 \\ \text { Density (calculated) } & 0.36 \times 0.14 \times 0.12 \mathrm{~mm}^{3} \\ \text { Absorption coefficient } & 7.1 \text { to } 134.11^{\circ} \\ \text { F(000) } & -9 \leq \mathrm{h} \leq 10,-29 \leq \mathrm{k} \leq 29,-9 \leq 1 \leq 9 \\ \text { Crystal size } & 31928 \\ \text { Theta range for data collection } & 2828\left[\mathrm{Rint}^{3}=0.0798, \mathrm{Rsigma}=0.0280\right] \\ \text { Index ranges } & 2828 / 1 / 229 \\ \text { Reflections collected } & 1.051 \\ \text { Independent reflections } & \mathrm{R} 1=0.0528, \mathrm{wR} 2=0.1421 \\ \text { Data/restraints/parameters } & \mathrm{R} 1=0.0687, \mathrm{wR} 2=0.1509 \\ \text { Goodness-of-fit on } \mathrm{F}^{2} & 0.25 /-0.25 \mathrm{e} \AA^{-3} \\ \text { Final R indexes [I }>=2 \sigma(\mathrm{I})] & \\ \text { Final R indexes [all data] } & \\ \text { Largest diff. peak/hole } & \end{array}$




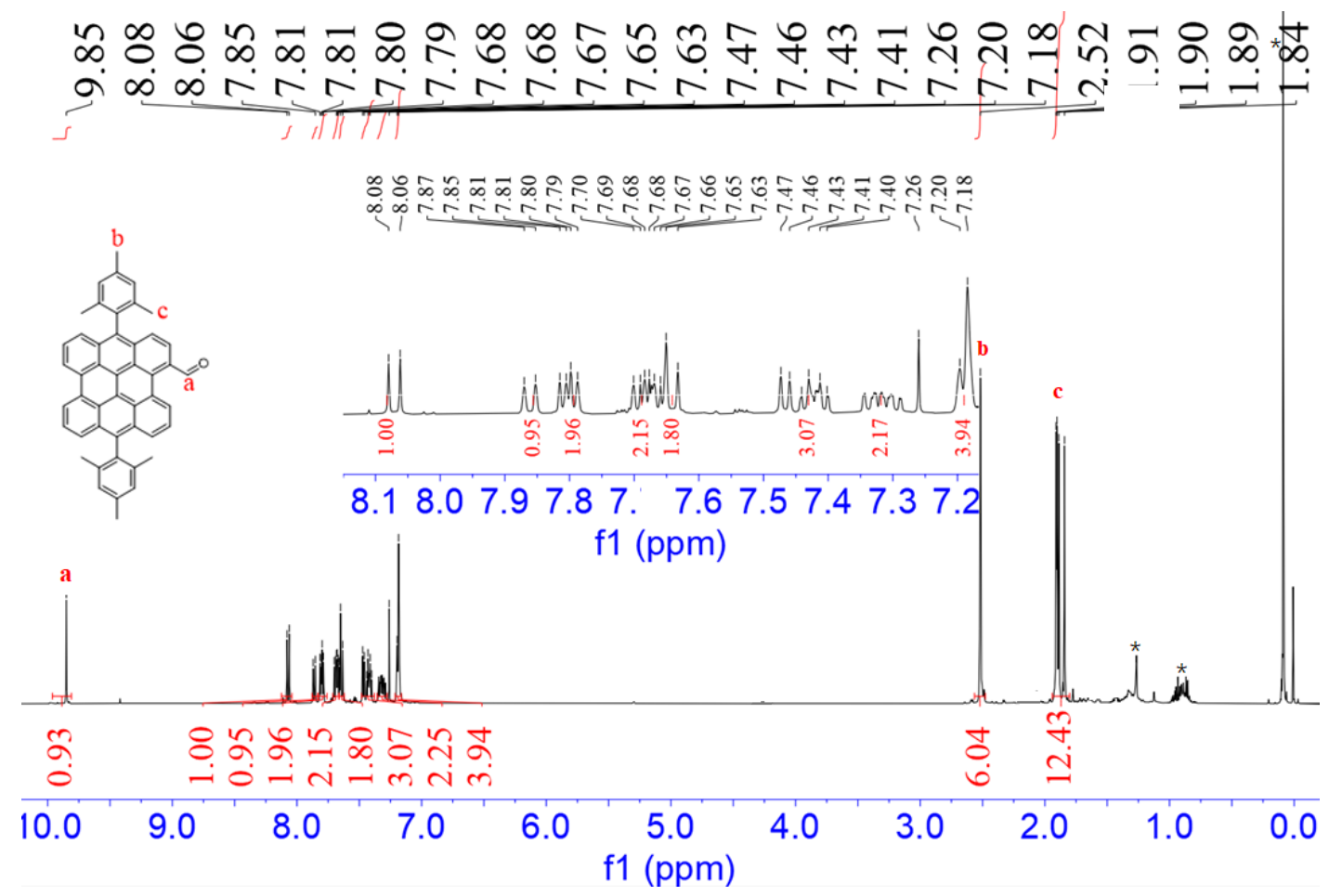

Figure S18. ${ }^{1} \mathrm{H}$ NMR $(500 \mathrm{MHz})$ spectrum of compound 1 in $\mathrm{CDCl}_{3}$ (* denotes the impurity from solvent).
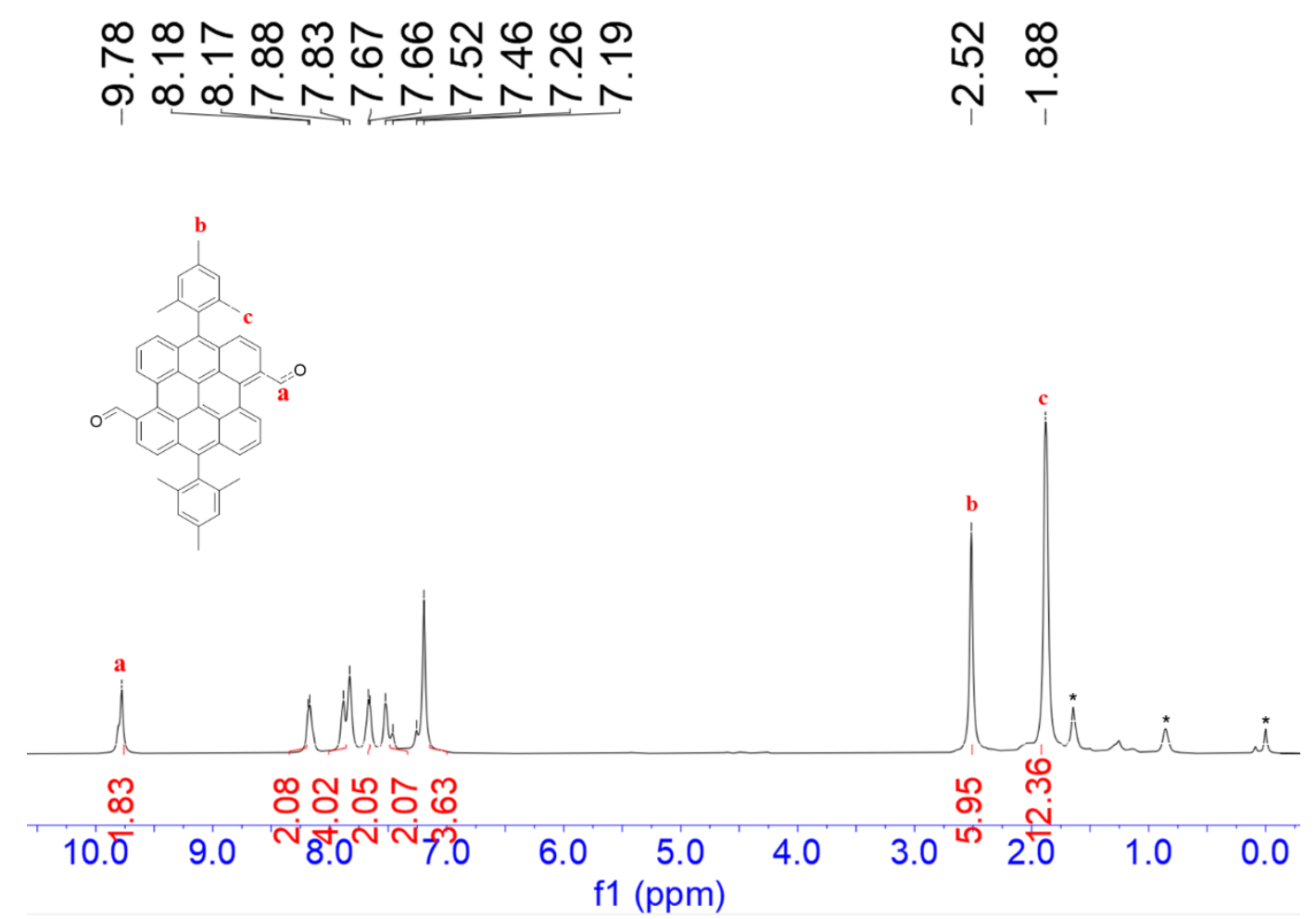

Figure S19. ${ }^{1} \mathrm{H}$ NMR (500 MHz) spectrum of compound 2 in $\mathrm{CDCl}_{3}$ (* denotes the impurity from solvent). 


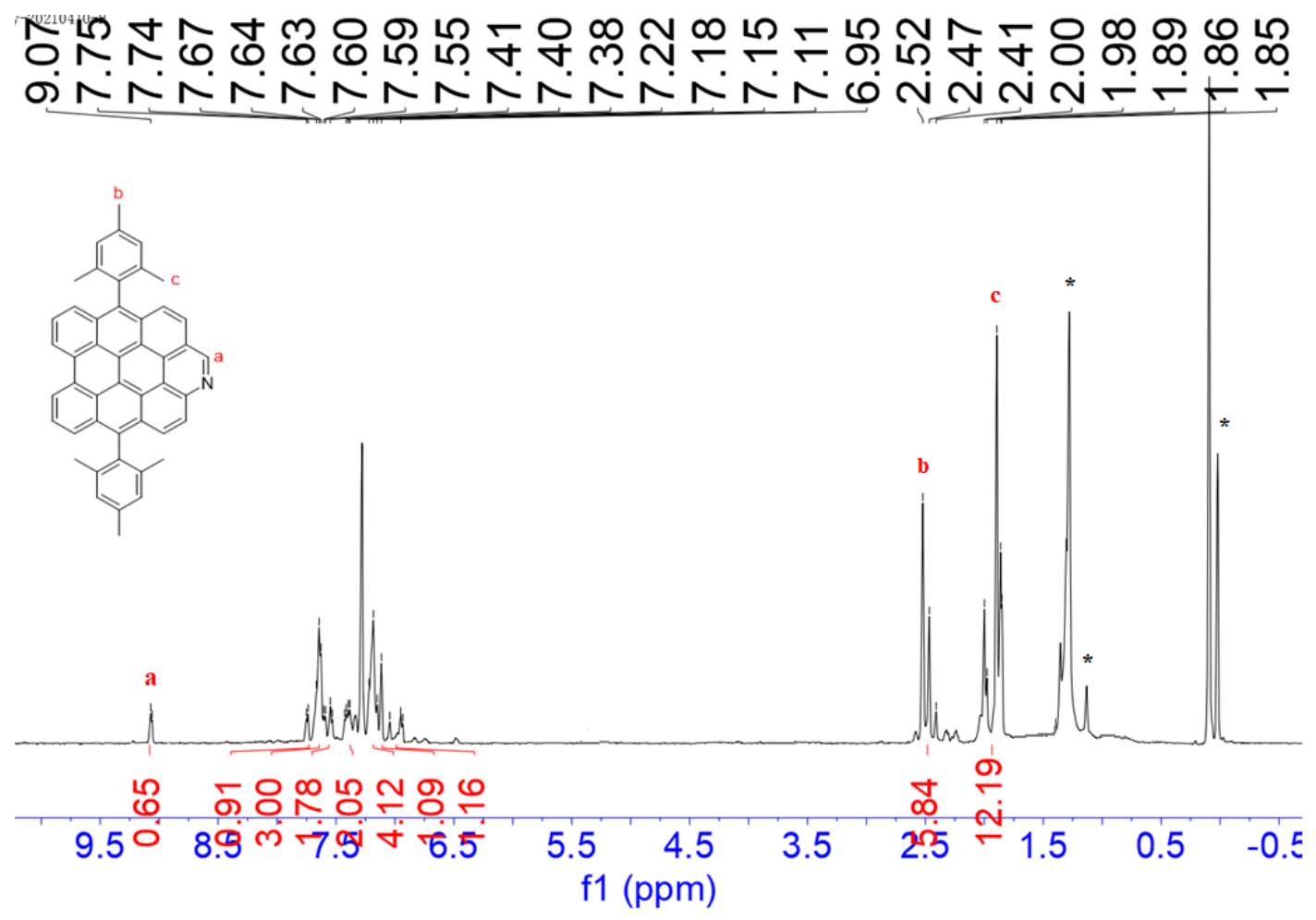

Figure S20. ${ }^{1} \mathrm{H}$ NMR $(500 \mathrm{MHz})$ spectrum of compound 3 in $\mathrm{CDCl}_{3}$ (* denotes the impurity from solvent).

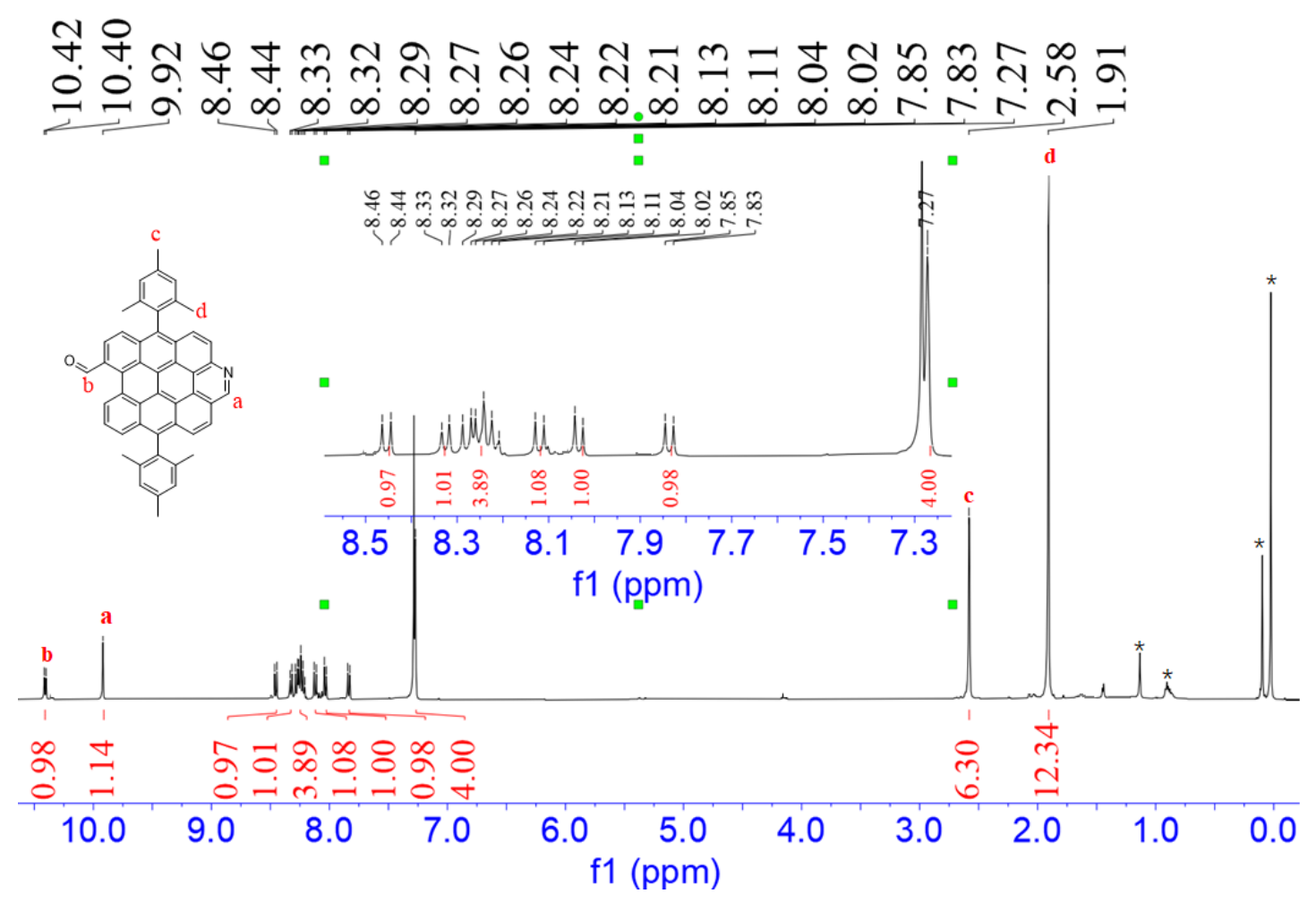

Figure S21. ${ }^{1} \mathrm{H}$ NMR $(500 \mathrm{MHz})$ spectrum of compound 4 in $\mathrm{CDCl}_{3}$ (* denotes the impurity from solvent). 


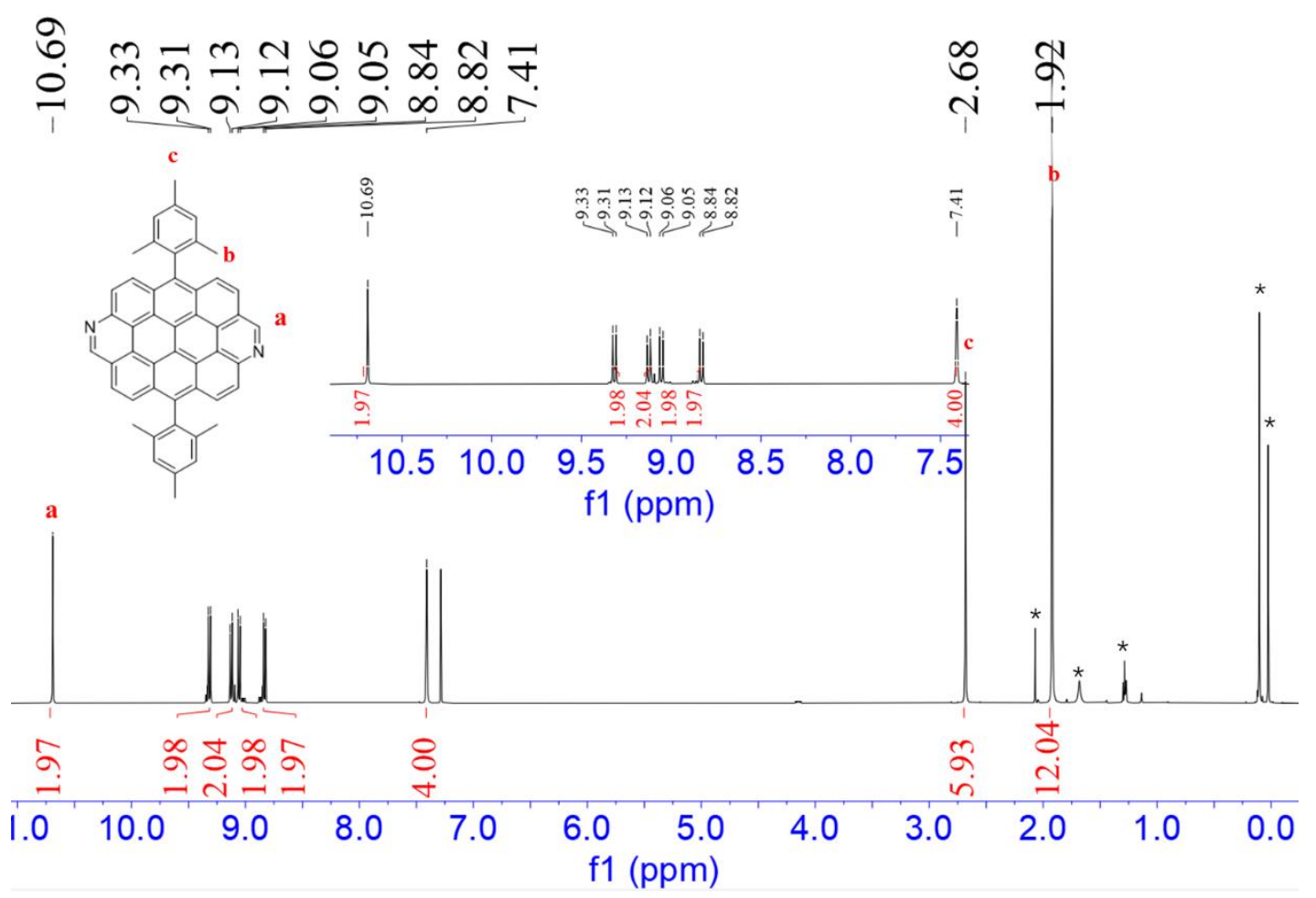

Figure S22. ${ }^{1} \mathrm{H}$ NMR $(500 \mathrm{MHz})$ spectrum of compound 5 in $\mathrm{CDCl}_{3}$ (* denotes the impurity from solvent).

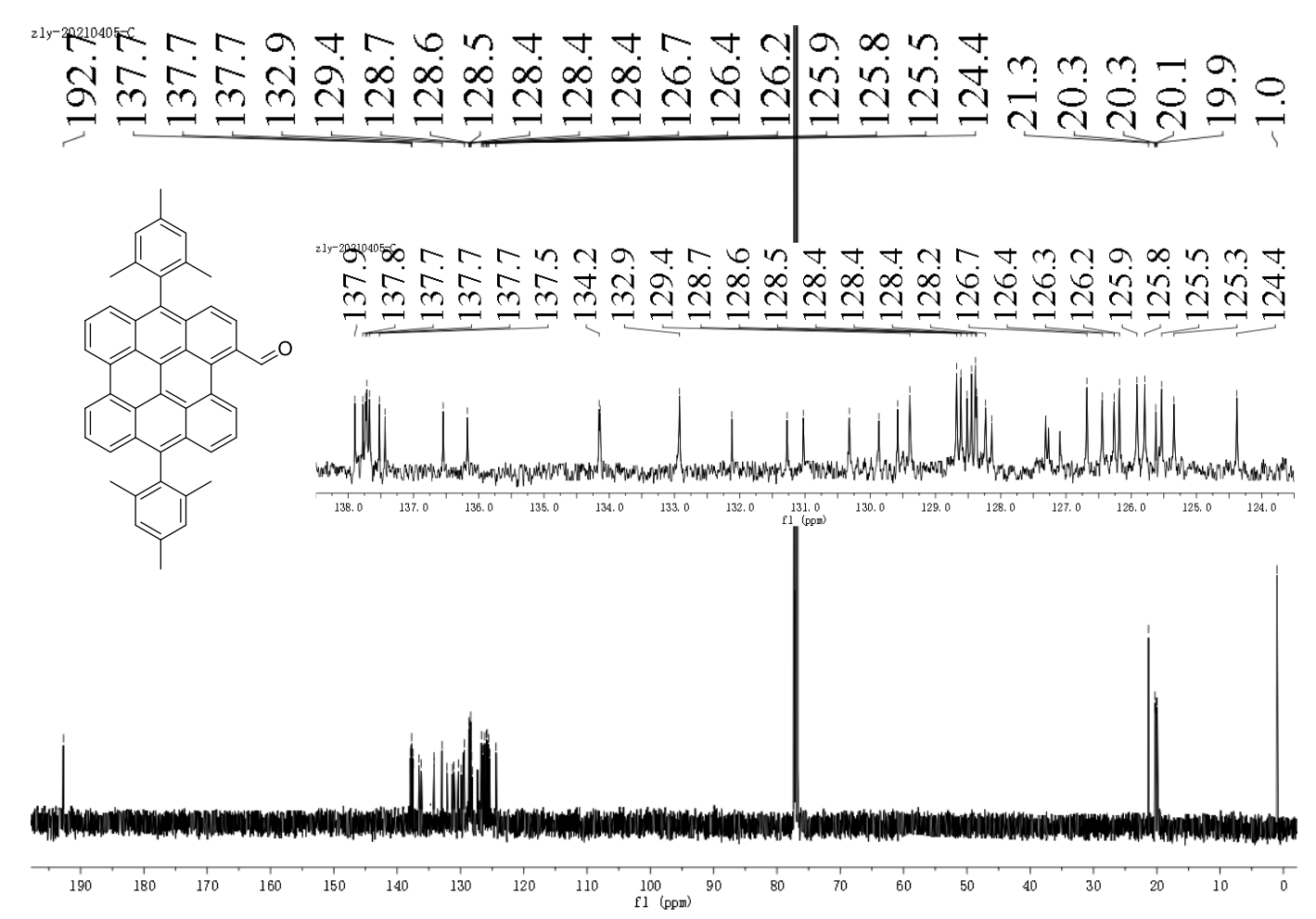

Figure S23. ${ }^{13} \mathrm{C}$ NMR (125 MHz) spectrum of compound 1 in $\mathrm{CDCl}_{3}$. 


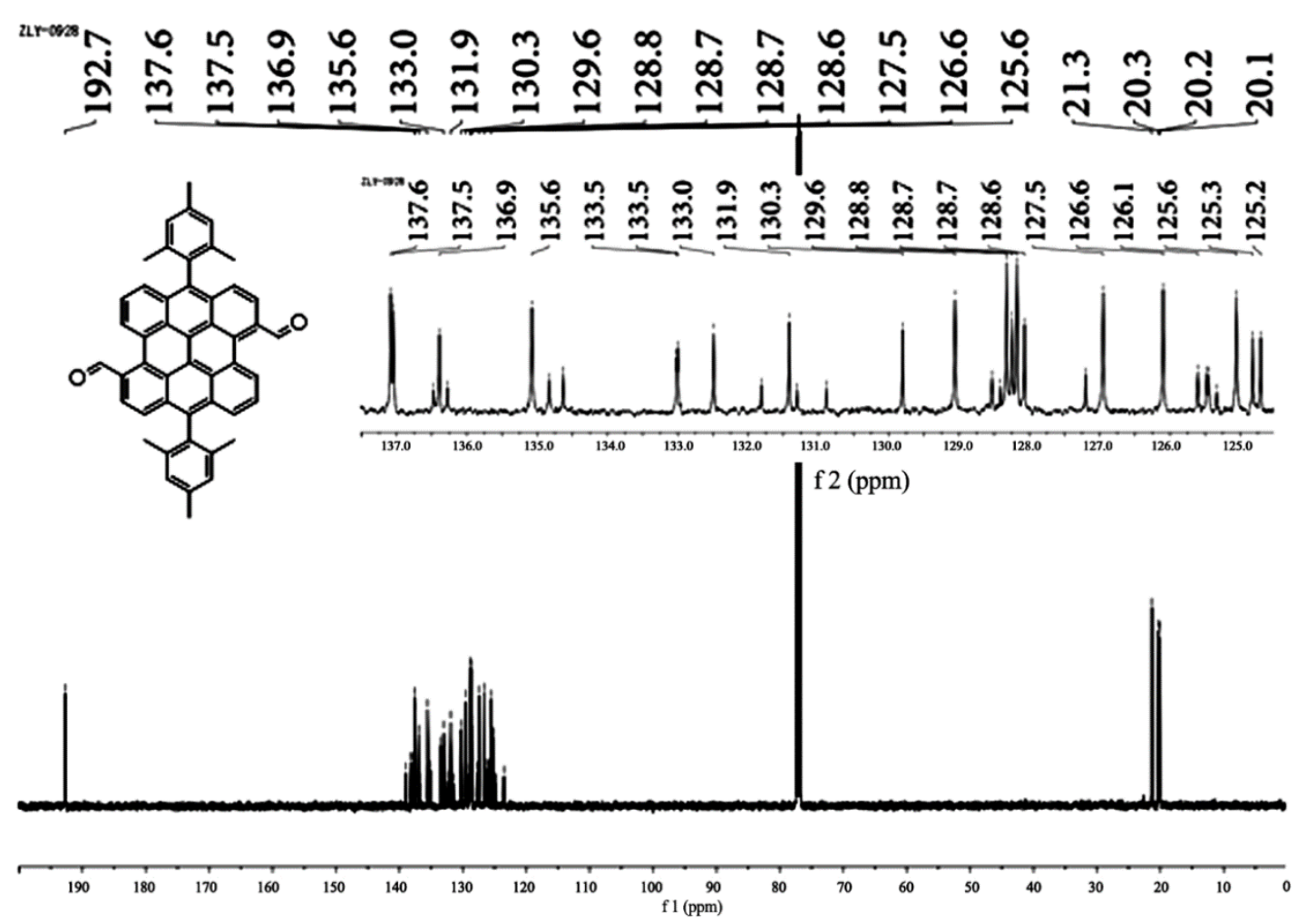

Figure S24. ${ }^{13} \mathrm{C}$ NMR $(125 \mathrm{MHz})$ spectrum of compound 2 in $\mathrm{CDCl}_{3}$.

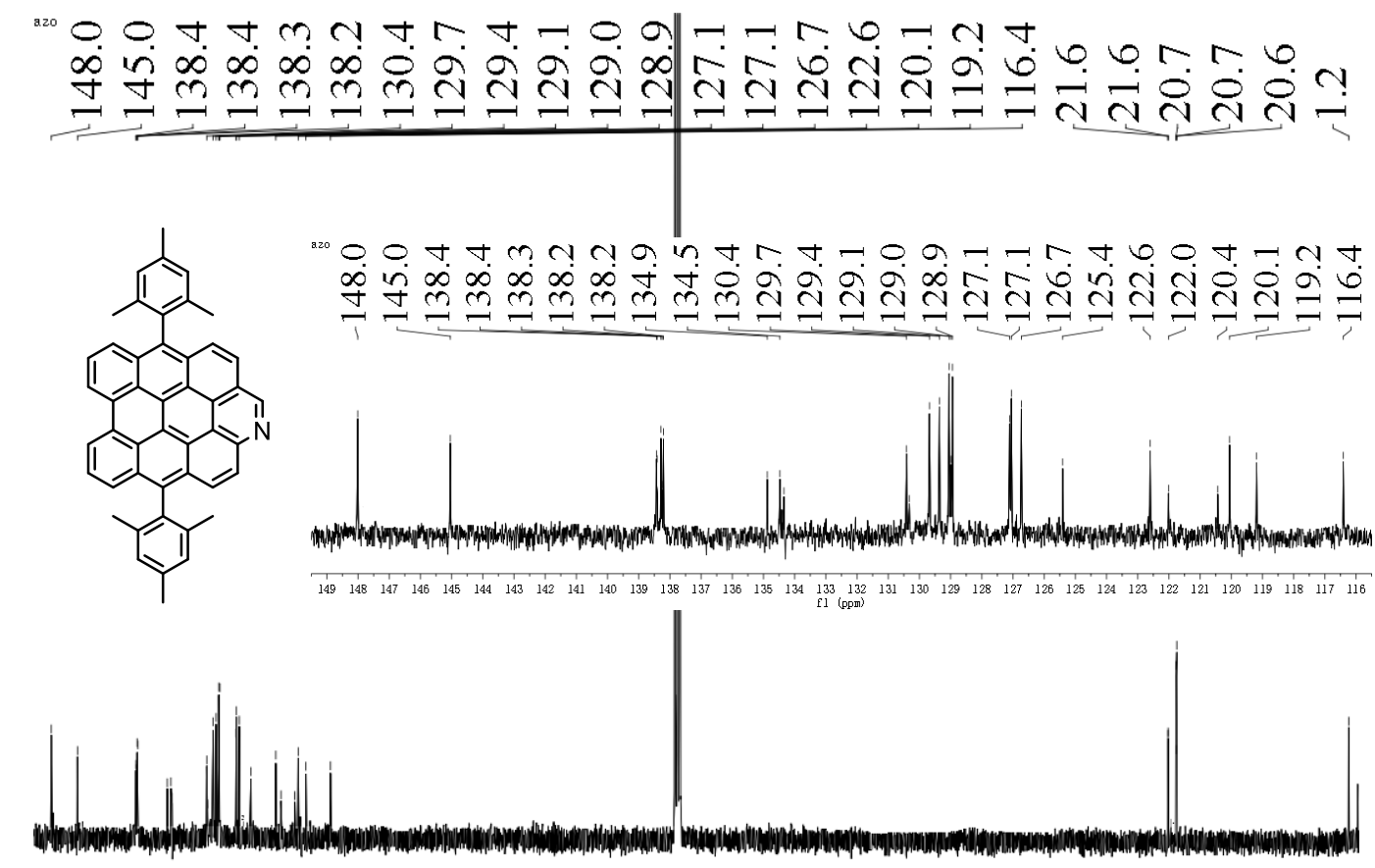

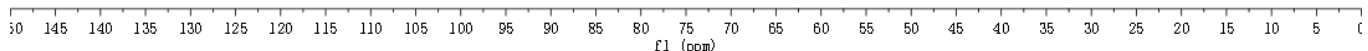

Figure S25. ${ }^{13} \mathrm{C}$ NMR (125 MHz) spectrum of compound 3 in $\mathrm{CDCl}_{3}$. 


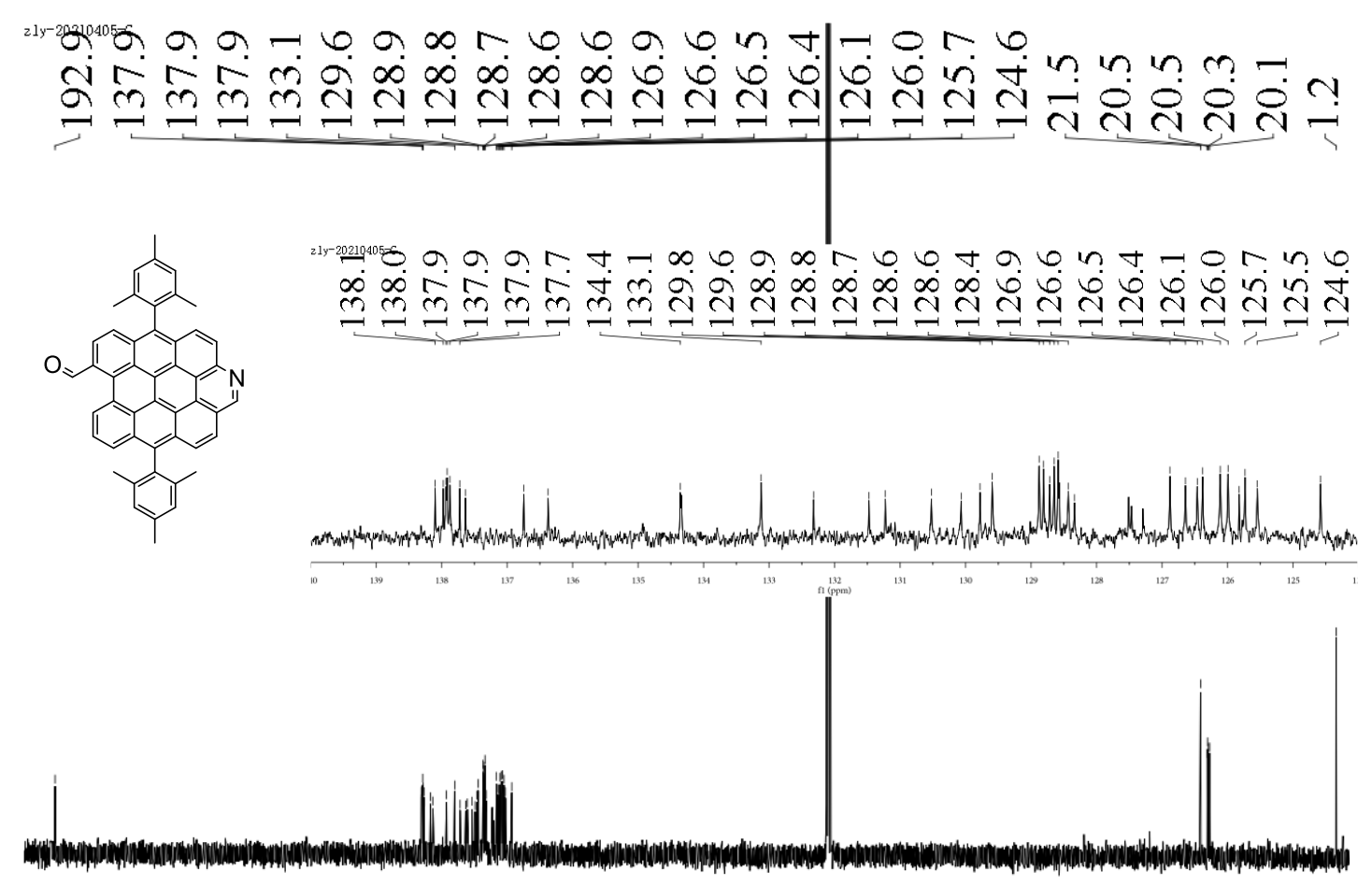

Figure S26. ${ }^{13} \mathrm{C}$ NMR (125 MHz) spectrum of compound 4 in $\mathrm{CDCl}_{3}$.

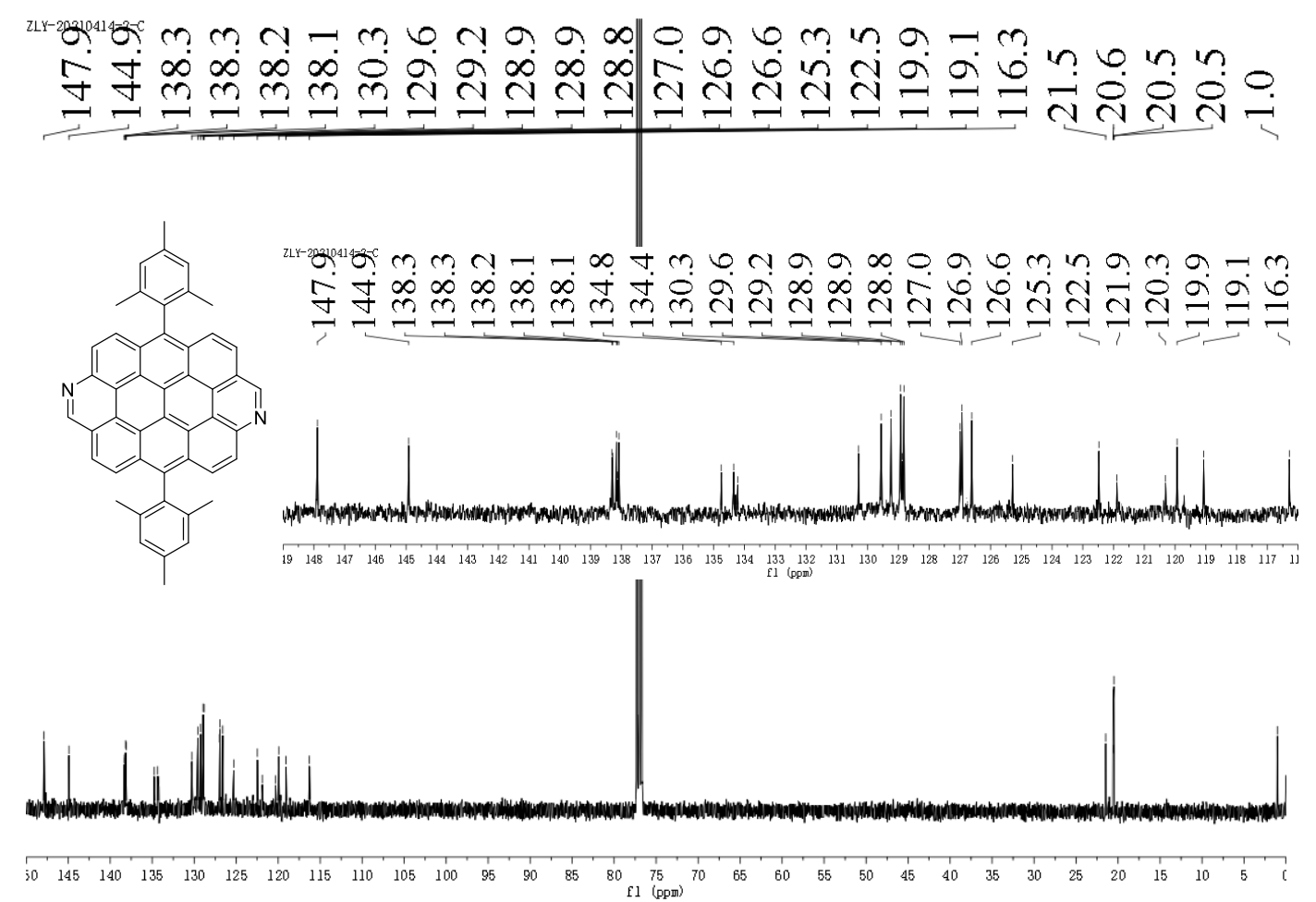

Figure S27. ${ }^{13} \mathrm{C}$ NMR (125 MHz) spectrum of compound 5 in $\mathrm{CDCl}_{3}$. 


\section{References}

S1. A. Konishi, Y. Hirao, K. Matsumoto, H. Kurata, T. Kubo, Chem. Lett. 2013, 42, 592-594.

S2. P. Thordarson, Chem. Soc. Rev. 2011, 40, 1305-1323.

S3. T. Lu, F. Chen, J. Comput. Chem., 2012, 33, 580-592.

S4. Z. Liu, T.Lu, Q. Chen, Carbon, 2020, 165, 461-467. 